\title{
Hopf Bifurcation and Singularity Induced Bifurcation in a Leslie-Gower Predator-Prey System with Nonlinear Harvesting
}

\author{
Wei Liu ${ }^{1,2}$ and Yaolin Jiang ${ }^{1, *}$ \\ ${ }^{1}$ School of Mathematics and Statistics, Xi'an Jiaotong University, \\ Xi'an 710049, China. \\ ${ }^{2}$ School of Mathematics and Computer Science, Xinyu University, \\ Xinyu 338004, China.
}

Received 3 May 2019; Accepted (in revised version) 11 July 2019.

\begin{abstract}
A modified predator-prey model with a new nonlinear harvesting on predator and gestation delay of prey is studied. It is shown that the stability of interior equilibrium point can switch finite times and Hopf bifurcations occur when delay increases through critical values. The properties of the Hopf bifurcations are investigated by the center manifold theorem. Special attention is paid to singularity-induced bifurcations and their state feedback control. Numerical simulations demonstrate the effectiveness of the theoretical results.
\end{abstract}

AMS subject classifications: 34D20, 34C23, 34C25, 92D25

Key words: Predator-prey, nonlinear harvesting, delay, Hopf bifurcation, singularity-induced bifurcation.

\section{Introduction and Preliminaries}

The dynamical relationship of predator-prey interaction is one of the most important research topics in mathematical biology [7, 13, 22]. In the 1920s, Vito Volterra and Alfred James Lotka established an ecosystem model for one predator population and one single prey population in a constant and uniform environment. This model, termed as standard Volterra-Lotka predator-prey model, is described by differential equations. It attracted considerable attention in applied mathematics and theoretical ecology. On the other hands, in population dynamics of predator-prey systems, a lot of efforts have been spent on applications of the dynamic theory of nonlinear differential equations $[7,12,13,22]$. However, it was discovered that within the Volterra-Lotka model, the growth rates of the predator and prey populations are not bounded, which contradicts real-world experience. Therefore, Leslie and Gower [23] proposed the following predator-prey model:

\footnotetext{
${ }^{*}$ Corresponding author. Email addresses: wliu2015@163.com (W. Liu), ylj iang@x j tu .edu .cn (Y.L. Jiang) 


$$
\begin{aligned}
& \dot{x}(t)=x(t)(a-x(t)-y(t)), \\
& \dot{y}(t)=y(t)\left(d-k \frac{y(t)}{x(t)}\right),
\end{aligned}
$$

where $x(t)$ and $y(t)$ are, respectively, the densities of prey and predator species, $a$ and $d$ the corresponding maximum growth rates of preys and predators, and the parameter $k>0$ represents the conversion rate from captured preys into predator births - cf. Refs. [7, 13, $22,23]$.

It is worth noting that in order to express the interaction and coexistence of biological populations, time delay is often incorporated in the predator-prey models depending on the past history. Generally speaking, it would induce a wealthier dynamical behavior, such as the loss of stability, oscillatory dynamics, various bifurcations (saddle-node, saddlenode-Hopf, Hopf-Andronov, Bogdanov-Takens), and chaos phenomenon [7, 12, 13, 21, 22, $29,37,39,42-44,46]$. Over the years, various predator-prey models with time delay have been studied [7, 13, 21, 22, 29, 43, 44]. One of such models - viz. predator-prey model with gestation delay is ubiquitous in the real world, which can be considered as inherent in biological populations. Taking into account Refs. $[7,12,13,22]$, we incorporate the gestation delay of prey population into the model (1.1), thus obtaining the following delayed predator-prey model

$$
\begin{aligned}
& \dot{x}(t)=x(t)(a-x(t-\tau)-y(t)), \\
& \dot{y}(t)=y(t)\left(d-k \frac{y(t)}{x(t)}\right) .
\end{aligned}
$$

In order to study the economic return from the harvesting effort of mankind on ecological resources, the following economic equation

$$
\text { Net Economic Revenue }=\text { Total Revenue }- \text { Total Cost }
$$

has been adopted $[8,14]$. Using the principle (1.3), we are going to study the economic revenue from the harvesting effort $E(t)$ on the predator population in the delayed predatorprey model (1.2). There are two common harvesting modes for predator-prey models viz. constant and proportional harvesting. The former means that the harvest rate is constant - i.e. it does not depend on population density, and the latter means that the higher number of preys or predators leads to a higher catch. The proportional harvesting is clear improvement to a constant harvesting. However, the harvest increase in the proportional harvesting can become unprofitable due to the market's oversupply.

Here, we propose a new type of nonlinear harvesting. Suppose that $T$ is the total time that each worker needs for harvesting. It usually includes the time $T_{1}$ required for looking of predators and the time $T_{2}$ for handling them. We also assume that the amount $N$ of predators caught by each worker is proportional to the searching time $T_{1}$ and to predators population density $y(t)$, i.e. $N=T_{1} y(t)$. Besides, $m$ is the average time spent by a worker on handling of each predator captured. Therefore, we have $T_{2}=m N=m T_{1} y(t)$, so that $T=T_{1}(1+m y(t))$. Hence, the amount of predators caught by a worker per unit time is $N / T=y(t) /(1+m y(t))$. This harvesting rate is adopted here and the number of predators 
captured by the workers of the harvesting company is $H(y(t))=y(t) E(t) /(1+m y(t))$. We observe that as the density of predators $y(t)$ grows, the amount of predators captured also grows but at a decreasing rate.

Furthermore, we let the positive parameters $p, c$ and $v$ be the harvest reward, the harvest cost and the net economic revenue, respectively. Combining it with the economic equation (1.3), we have

$$
\text { Total Revenue }=p H(t)=\frac{p y(t) E(t)}{1+m y(t)}, \quad \text { Total Cost }=c E(t) .
$$

Consequently, the net economic revenue $v$ is

$$
v=E(t)\left(\frac{p y(t)}{1+m y(t)}-c\right) .
$$

Taking into account the Eqs. (1.2),(1.4), we consider the following modified predator-prey model with delay and nonlinear harvesting:

$$
\begin{aligned}
& \dot{x}(t)=x(t)(a-x(t-\tau)-y(t)), \\
& \dot{y}(t)=y(t)\left(d-k \frac{y(t)}{x(t)}-\frac{E(t)}{1+m y(t)}\right), \\
& 0=E(t)\left(\frac{p y(t)}{1+m y(t)}-c\right)-v
\end{aligned}
$$

with the harvesting effort $E(t)$ viewed as a variable in the dynamical analysis below.

Most of conventional predator-prey models with harvesting are formulated by differential equations $[8,16,19,21]$. However, in our case the harvested predator-prey model (1.5) also contains the algebraic equation (1.4) coupled with the system (1.2). It is clear that in a highly-developed commodity economy, harvested biological resources can be sold for economical benefits. Thus unlike the traditional harvested predator-prey models $[8,16,19,21]$, our approach includes not only predator-prey interactions but also the harvesting on biological population from economic viewpoint. Several modified predator-prey models related to the model (1.5) have been considered in literature so far - cf. [5, 24-26, 44, 45]. In particular, Zhang et al. [44] used the reduction method for retarded functional differential equations [11] to study the Hopf bifurcation in a harvested predator-prey model with maturation and digestion delays of the predator population. The work [26] employs results of [9] and the theory of functional differential equations [17], to investigate the Hopf bifurcation in a Gause predator-prey model with proportional harvesting and time lag. The stability, oscillations, periodic motion and the properties of flip bifurcations and Neimark-Sacker bifurcations around the interior equilibria for discrete predator-prey models with harvesting are considered in [5,45]. Li et al. [24] exploited Lyapunov stability theory and Hopf bifurcation theorem $[15,28]$ to study equilibria in an ordinary harvested predator-prey model with Holling type II response function. The conditions for Hopf bifurcation and stability of Hopf bifurcating periodic solutions in an ordinary predator-prey model with Holling type IV response function is studied in [25] by the formal series method from [40,41]. 
Nevertheless, various problems concerning the bifurcating periodic orbits in delayed differential-algebraic predator-prey systems with nonlinear harvesting remain open. Considering a new type of bifurcation - viz. a singularity-induced bifurcation phenomenon, we construct a corresponding state feedback controller to eliminate the bifurcation. A singularity-induced bifurcation describes the spectral divergence through infinity [32]. Singularity-induced bifurcations in differential-algebra systems represent a challenging problem and produce effects not appearing in systems described by differential equations [1,27,32]. In the model (1.5) we have typical Hopf and singularity-induced bifurcations. In the latter, the equilibrium point is placed at a singular surface. To the best of author's knowledge, singularity-induced bifurcation of biological systems with the nonlinear harvesting have not been yet studied. In our considerations, we use center manifold theory [18], parameterisation method [6], singularity-induced bifurcation theory [35] and singularity control theory for differential-algebra equations [10] different from [5, 8, 16, 19, 21, 24-26, 44, 45].

This work complements the investigations $[5,8,16,19,21,24-26,44,45]$ concerning the dynamical modelling of predator-prey models. In particular, we rely on analytical methods of differential-algebraic equations widely used in nonlinear dynamical control systems cf. $[1,20,27,31,32,35]$, so that the present paper can be considered as an application of the differential-algebra system theory in biology.

Let us now recall some basic facts needed in what follows.

If $v>0$, then according to [28], $X_{0}=\left(x_{0}, y_{0}, E_{0}\right)^{T}$ is an equilibrium point for the model (1.5) if and only if

$$
\begin{aligned}
& a-x_{0}-y_{0}=0, \\
& d-k \frac{y_{0}}{x_{0}}-\frac{E_{0}}{1+m y_{0}}=0, \\
& E_{0}\left(\frac{p y_{0}}{1+m y_{0}}-c\right)-v=0 .
\end{aligned}
$$

Therefore, (1.5) has the equilibrium point

$$
X_{0}=\left(x_{0}, y_{0}, E_{0}\right)^{T}=\left(a-y_{0}, y_{0}, \frac{\left(1+m y_{0}\right) v}{(p-c m) y_{0}-c}\right)^{T},
$$

where $y_{0}$ satisfies

$$
(d+k)(p-c m) y_{0}^{2}-(a+c d+c k+a d p-a c d m) y_{0}+a(v+c d)=0 .
$$

Moreover, it follows from $[20,31]$ that the model $(1.5)$ is locally equivalent to the following differential-algebra system around its equilibrium point $X_{0}$ :

$$
\begin{aligned}
& \dot{x}(t)=x(t)(a-x(t-\tau)-y(t)), \\
& \dot{y}(t)=y(t)\left(d-k \frac{y(t)}{x(t)}-\frac{E(t)}{1+m y(t)}\right), \\
& \dot{E}(t)=f_{3}(x(t), y(t), E(t)), \\
& 0=E(t)\left(\frac{p y(t)}{1+m y(t)}-c\right)-v,
\end{aligned}
$$


where $f_{3}(x, y, E)$ is a continuously differentiable function such that $f_{3}\left(x_{0}, y_{0}, E_{0}\right)=0$. An explicit representation for $f_{3}(x, y, E)$ is not needed. We write

$$
\begin{aligned}
& f(X):=\left(\begin{array}{c}
f_{1}(X) \\
f_{2}(X) \\
f_{3}(X)
\end{array}\right)=\left(\begin{array}{c}
x(t)(a-x(t-\tau)-y(t)) \\
y(t)\left(d-k \frac{y(t)}{x(t)}-\frac{E(t)}{1+m y(t)}\right) \\
f_{3}(x(t), y(t), E(t))
\end{array}\right) \\
& g(X):=E(t)\left(\frac{p y(t)}{1+m y(t)}-c\right)-v, \quad X=(x, y, E)^{T}
\end{aligned}
$$

and omit $t$ if it will cause no confusion.

\section{Local Stability and Hopf Bifurcations}

According to $[7,13,22]$, the equilibrium point $X_{0}$ is an interior point - i.e. $x_{0}, y_{0}$ and $E_{0}$ should be positive. This means that the populations of predators and preys and harvesting efforts are exist. Therefore, here and in what follows we always assume that

$$
p-c m>0, \quad K_{1}>0, \quad K_{1}^{2}-4 K_{2} \geq 0, \quad y_{0}<a, \quad v>0, \quad(p-c m) y_{0}>c,
$$

where

$$
\begin{aligned}
& K_{1}=\frac{a+c d+c k+a d p-a c d m}{[(d+k)(p-c m)]}, \\
& K_{2}=\frac{a(v+c d)}{[(d+k)(p-c m)]} .
\end{aligned}
$$

Considering the system (1.6), we find out that

$$
D_{X} g\left(X_{0}\right)=\left(0, \frac{p E_{0}}{\left(1+m y_{0}\right)^{2}}, \frac{p y_{0}}{\left(1+m y_{0}\right)}-c\right),
$$

where $D_{X} g(X)$ is the Jacobi matrix of $g(X)$ with respect to $X$. Besides, let

$$
\begin{aligned}
\bar{X}(t) & :=(x(t), y(t), \bar{E}(t))^{T}, \\
Q & :=\left(\begin{array}{lll}
1 & 0 & 0 \\
0 & 1 & 0 \\
0 & -\frac{p E_{0}}{\left(1+m y_{0}\right)\left[(p-c m) y_{0}-c\right]} & 1
\end{array}\right) .
\end{aligned}
$$

In order to use the parameterisation method of Appendix A, we apply the nonsingular transformation $X=Q \bar{X}$ to the system (1.6), thus obtaining

$$
\begin{aligned}
& \dot{x}(t)=x(t)(a-x(t-\tau)-y(t)), \\
& \dot{y}(t)=y(t)\left(d-k \frac{y(t)}{x(t)}+\frac{p E_{0} y(t)}{\left(1+m y_{0}\right)\left[(p-c m) y_{0}-c\right](1+m y(t))}-\frac{\bar{E}(t)}{1+m y(t)}\right), \\
& \dot{\bar{E}}(t)=f_{3}(x(t), y(t), \bar{E}(t)), \\
& 0=\left(-\frac{p E_{0} y(t)}{\left(1+m y_{0}\right)\left[(p-c m) y_{0}-c\right]}+\bar{E}(t)\right)\left(\frac{p y(t)}{1+m y(t)}-c\right)-v,
\end{aligned}
$$


where $f_{3}(x, y, \bar{E})$ is a continuously differentiable function such that $f_{3}\left(\bar{X}_{0}\right)=0$ at the equilibrium point

$$
\bar{X}_{0}=\left(x_{0}, y_{0}, \bar{E}_{0}\right)^{T}=\left(x_{0}, y_{0}, E_{0}+\frac{p y_{0} E_{0}}{\left\{\left(1+m y_{0}\right)\left[(p-c m) y_{0}-c\right]\right\}}\right)^{T}
$$

of the system (2.2). As was already mentioned, the specific form of $f_{3}(x, y, \bar{E})$ is not important - cf. Eqs. (2.5) below. Recalling the notation of Section 1, we write

$$
\begin{aligned}
& f(\bar{X}):=\left(\begin{array}{c}
x(t)(a-x(t-\tau)-y(t)) \\
y(t)\left(d-k \frac{y(t)}{x(t)}+\frac{p E_{0} y(t)}{\left(1+m y_{0}\right)\left[(p-c m) y_{0}-c\right](1+m y(t))}-\frac{\bar{E}(t)}{1+m y(t)}\right) \\
f_{3}(x(t), y(t), \bar{E}(t))
\end{array}\right), \\
& g(\bar{X}):=\left(-\frac{p E_{0} y(t)}{\left(1+m y_{0}\right)\left[(p-c m) y_{0}-c\right]}+\bar{E}(t)\right)\left(\frac{p y(t)}{1+m y(t)}-c\right)-v,
\end{aligned}
$$

where $f(\bar{X}):=\left(f_{1}(\bar{X}), f_{2}(\bar{X}), f_{3}(\bar{X})\right)^{T}$. It follows that

$$
\begin{aligned}
D_{\bar{X}} g\left(\bar{X}_{0}\right)= & \left(0, \frac{p}{\left(1+m y_{0}\right)^{2}}\left[E_{0}+\frac{p y_{0} E_{0}}{\left(1+m y_{0}\right)\left[(p-c m) y_{0}-c\right]}\right]\right. \\
& \left.-\frac{p^{2} y_{0} E_{0}}{\left(1+m y_{0}\right)^{3}\left[(p-c m) y_{0}-c\right]}-\frac{p E_{0}}{\left(1+m y_{0}\right)^{2}}, \frac{(p-c m) y_{0}-c}{1+m y_{0}}\right) \\
= & \left(0,0, \frac{(p-c m) y_{0}-c}{1+m y_{0}}\right) .
\end{aligned}
$$

It is easily seen that $\operatorname{rank} D_{\bar{X}} g\left(\bar{X}_{0}\right)=1$ and the system (2.2) satisfies the conditions of the parameterisation method of Appendix A. Following $[4,6]$, we will use the following parameterisation $\psi$ for (2.2):

$$
\bar{X}(t)=\psi(Y(t))=\bar{X}_{0}+U_{0} Y(t)+V_{0} h(Y(t)) \text { and } \quad g(\psi(Y(t)))=0,
$$

where

$$
Y(t):=\left(y_{1}(t), y_{2}(t)\right)^{T} \in \mathbb{R}^{2}, \quad U_{0}=\left(\begin{array}{ll}
1 & 0 \\
0 & 1 \\
0 & 0
\end{array}\right), \quad V_{0}=\left(\begin{array}{l}
0 \\
0 \\
1
\end{array}\right),
$$

and $h(Y(t))$ is a smooth mapping from $\mathbb{R}^{2}$ to $\mathbb{R}$, the existence of which is guaranteed by the implicit function theorem [4]. Taking into account the Eq. (A.8), we write the Taylor expansions for the parameterised system of (2.2) as

$$
\dot{Y}(t)=U_{0}^{T} D_{\bar{X}} f\left(\bar{X}_{0}\right)\left(\begin{array}{c}
D_{\bar{X}} g\left(\bar{X}_{0}\right) \\
U_{0}^{T}
\end{array}\right)^{-1}\left(\begin{array}{c}
0 \\
I_{2}
\end{array}\right) Y(t)+\mathscr{O}(|Y|) .
$$


Note that since

$$
\bar{E}_{0}=E_{0}+\frac{p y_{0} E_{0}}{\left\{\left(1+m y_{0}\right)\left[(p-c m) y_{0}-c\right]\right\}},
$$

the Jacobi matrix of the linearised system (2.4) has the form

$$
\begin{aligned}
& U_{0}^{T} D_{\bar{X}} f\left(\bar{X}_{0}\right)\left(\begin{array}{c}
D_{\bar{X}} g\left(\bar{X}_{0}\right) \\
U_{0}^{T}
\end{array}\right)^{-1}\left(\begin{array}{c}
0 \\
I_{2}
\end{array}\right) \\
= & \left(\begin{array}{c}
D_{\bar{X}} f_{1}\left(\bar{X}_{0}\right) \\
D_{\bar{X}} f_{2}\left(\bar{X}_{0}\right)
\end{array}\right)\left(\begin{array}{c}
D_{\bar{X}} g\left(\bar{X}_{0}\right) \\
U_{0}^{T}
\end{array}\right)^{-1}\left(\begin{array}{c}
0 \\
I_{2}
\end{array}\right)=\left(\begin{array}{cc}
-x_{0} e^{-\lambda \tau} & -x_{0} \\
\frac{k y_{0}^{2}}{x_{0}^{2}} & -\mathscr{M}
\end{array}\right),
\end{aligned}
$$

where

$$
\mathscr{M}=\frac{k y_{0}}{x_{0}}-\frac{m y_{0} E_{0}}{\left(1+m y_{0}\right)^{2}}-\frac{p y_{0} E_{0}}{\left(1+m y_{0}\right)^{2}\left[(p-c m) y_{0}-c\right]} .
$$

The terms $f_{3}(x, y, E)$ and $f_{3}(x, y, \bar{E})$ are absent here, so that they are not needed in what follows. The characteristic equation for the Jacobi matrix (2.5) is

$$
\lambda^{2}+\left(x_{0} e^{-\lambda \tau}+\mathscr{M}\right) \lambda+\frac{k y_{0}^{2}}{x_{0}}+x_{0} \mathscr{M} e^{-\lambda \tau}=0
$$

and for $\tau=0$ it takes the form

$$
\lambda^{2}+\left(x_{0}+\mathscr{M}\right) \lambda+\frac{k y_{0}^{2}}{x_{0}}+x_{0} \mathscr{M}=0 .
$$

The following lemma is a consequence of the Routh-Hurwitz criteria [34].

Lemma 2.1. For model (1.5) with $\tau=0$, we have:

(i) If

$$
\begin{aligned}
& x_{0}+\frac{k y_{0}}{x_{0}}>\frac{m y_{0} E_{0}}{\left(1+m y_{0}\right)^{2}}+\frac{p y_{0} E_{0}}{\left(1+m y_{0}\right)^{2}\left[(p-c m) y_{0}-c\right]}, \\
& k+\frac{k y_{0}}{x_{0}}>\frac{m x_{0} E_{0}}{\left(1+m y_{0}\right)^{2}}+\frac{p x_{0} E_{0}}{\left(1+m y_{0}\right)^{2}\left[(p-c m) y_{0}-c\right]},
\end{aligned}
$$

then the interior equilibrium point $X_{0}$ of the model (1.5) is locally asymptotically stable.

(ii) If

$$
x_{0}+\frac{k y_{0}}{x_{0}}<\frac{m y_{0} E_{0}}{\left(1+m y_{0}\right)^{2}}+\frac{p y_{0} E_{0}}{\left(1+m y_{0}\right)^{2}\left[(p-c m) y_{0}-c\right]},
$$

then the interior equilibrium point $X_{0}$ of the model (1.5) is unstable. 
(iii) If

$$
\begin{aligned}
& x_{0}+\frac{k y_{0}}{x_{0}}=\frac{m y_{0} E_{0}}{\left(1+m y_{0}\right)^{2}}+\frac{p y_{0} E_{0}}{\left(1+m y_{0}\right)^{2}\left[(p-c m) y_{0}-c\right]} \\
& k+\frac{k y_{0}}{x_{0}}>\frac{m x_{0} E_{0}}{\left(1+m y_{0}\right)^{2}}+\frac{p x_{0} E_{0}}{\left(1+m y_{0}\right)^{2}\left[(p-c m) y_{0}-c\right]}
\end{aligned}
$$

then the interior equilibrium point $X_{0}$ of the model (1.5) is a center.

Remark 2.1. In case (iii), the Eq. (2.7) has purely imaginary roots, and for $\tau=0$ the model (1.5) may have Hopf bifurcation. To clarify the situation, one can use the Hopf bifurcation theorem [15].

For $\tau>0$, let $\lambda=i \omega, \omega>0$ be a root of the Eq. (2.6). Substituting it into the Eq. (2.6), we obtain

$$
-\omega^{2}+\left[x_{0}(\cos \omega \tau-i \sin \omega \tau)+\mathscr{M}\right] i \omega+\frac{k y_{0}^{2}}{x_{0}}+x_{0} \mathscr{M}(\cos \omega \tau-i \sin \omega \tau)=0,
$$

and the separation of real and imaginary parts yields

$$
\begin{aligned}
& \omega x_{0} \sin \omega \tau+x_{0} \mathscr{M} \cos \omega \tau=\omega^{2}-\frac{k y_{0}^{2}}{x_{0}}, \\
& \omega x_{0} \cos \omega \tau-x_{0} \mathscr{M} \sin \omega \tau=-\omega \mathscr{M} .
\end{aligned}
$$

It follows from the Eqs. (2.8),(2.9) that

$$
\cos \omega \tau=-\frac{k y_{0}^{2} \mathscr{M}}{\omega^{2} x_{0}^{2}+x_{0}^{2} \mathscr{M}^{2}},
$$

and

$$
\omega^{4}+\left(\mathscr{M}^{2}-x_{0}^{2}-\frac{2 k y_{0}^{2}}{x_{0}}\right) \omega^{2}+\frac{k^{2} y_{0}^{4}}{x_{0}^{2}}-x_{0}^{2} \mathscr{M}^{2}=0 .
$$

Lemma 2.2. If $\tau>0$, then we have:

(i) If

$$
\begin{aligned}
& x_{0}+\frac{k y_{0}}{x_{0}}>\frac{m y_{0} E_{0}}{\left(1+m y_{0}\right)^{2}}+\frac{p y_{0} E_{0}}{\left(1+m y_{0}\right)^{2}\left[(p-c m) y_{0}-c\right]} \\
& k+\frac{k y_{0}}{x_{0}}>\frac{m x_{0} E_{0}}{\left(1+m y_{0}\right)^{2}}+\frac{p x_{0} E_{0}}{\left(1+m y_{0}\right)^{2}\left[(p-c m) y_{0}-c\right]} \\
& \mathscr{M}^{2}>x_{0}^{2}+\frac{2 k y_{0}^{2}}{x_{0}}
\end{aligned}
$$

then the real parts of roots of the Eq. (2.6) are negative. 
(ii) If the term

$$
\widehat{\Delta}=\left(\mathscr{M}^{2}-x_{0}^{2}-\frac{2 k y_{0}^{2}}{x_{0}}\right)^{2}-\frac{4 k^{2} y_{0}^{4}}{x_{0}^{2}}+4 x_{0}^{2} \mathscr{M}^{2}
$$

is positive and

$$
\begin{aligned}
& \mathscr{M}^{2}<x_{0}^{2}+\frac{2 k y_{0}^{2}}{x_{0}}, \\
& \frac{k^{2} y_{0}^{2}}{x_{0}^{2}}>\left(k-\frac{m x_{0} E_{0}}{\left(1+m y_{0}\right)^{2}}-\frac{p x_{0} E_{0}}{\left(1+m y_{0}\right)^{2}\left[(p-c m) y_{0}-c\right]}\right)^{2},
\end{aligned}
$$

then the roots $\omega_{+}$and $\omega_{-}$of the Eq. (2.11) are positive. Substituting them into the Eq. (2.10), we obtain

$$
\tau_{\mathscr{K}}^{ \pm}=\frac{2 \mathscr{K} \pi}{\omega_{ \pm}}+\frac{1}{\omega_{ \pm}} \arccos \left(-\frac{k y_{0}^{2} \mathscr{M}}{\omega_{ \pm}^{2} x_{0}^{2}+x_{0}^{2} \mathscr{M}^{2}}\right),
$$

where $\mathscr{K}=0,1,2, \ldots$

Proof. Considering the polynomial equation (2.11), we note that if

$$
\mathscr{M}^{2}>x_{0}^{2}+\frac{2 k y_{0}^{2}}{x_{0}},
$$

then it does not have positive roots. Subsequently, the Eq. (2.6) does not have purely imaginary roots. Furthermore, if

$$
\begin{aligned}
& x_{0}+\frac{k y_{0}}{x_{0}}>\frac{m y_{0} E_{0}}{\left(1+m y_{0}\right)^{2}}+\frac{p y_{0} E_{0}}{\left(1+m y_{0}\right)^{2}\left[(p-c m) y_{0}-c\right]}, \\
& k+\frac{k y_{0}}{x_{0}}>\frac{m x_{0} E_{0}}{\left(1+m y_{0}\right)^{2}}+\frac{p x_{0} E_{0}}{\left(1+m y_{0}\right)^{2}\left[(p-c m) y_{0}-c\right]},
\end{aligned}
$$

then the real parts of the roots of the Eq. (2.7) are negative. By Rouche's theorem [33], the roots of the Eq. (2.6) have negative real parts as well.

Examining the case (ii), we note that if $\widehat{\Delta}>0$ and conditions (2.12)-(2.13) hold, then the polynomial equation (2.11) has two positive roots - viz.

$$
\omega_{ \pm}=\left\{\frac{x_{0}^{2}+\left(2 k y_{0}^{2}\right) / x_{0}-\mathscr{M}^{2} \pm \sqrt{\widehat{\Delta}}}{2}\right\}^{1 / 2} .
$$

Substituting $\omega_{ \pm}$into (2.10) and solving it with respect to $\tau$, we complete the proof.

Differentiating the Eq. (2.6) in $\tau$ yields

$$
2 \lambda \frac{\mathrm{d} \lambda}{\mathrm{d} \tau}+\mathscr{M} \frac{\mathrm{d} \lambda}{\mathrm{d} \tau}+\lambda x_{0} e^{-\lambda \tau}\left(-\lambda-\tau \frac{\mathrm{d} \lambda}{\mathrm{d} \tau}\right)+x_{0} e^{-\lambda \tau} \frac{\mathrm{d} \lambda}{\mathrm{d} \tau}+x_{0} \mathscr{M} e^{-\lambda \tau}\left(-\lambda-\tau \frac{\mathrm{d} \lambda}{\mathrm{d} \tau}\right)=0
$$


so that

$$
\left(\frac{\mathrm{d} \lambda}{\mathrm{d} \tau}\right)^{-1}=\frac{2 \lambda e^{\lambda \tau}+\mathscr{M}\left(e^{\lambda \tau}-\tau x_{0}\right)+x_{0}-\lambda \tau x_{0}}{\lambda^{2} x_{0}+\lambda x_{0} \mathscr{M}} .
$$

Substituting $\lambda=i \omega$ into Eq. (2.14), we obtain

$$
\begin{aligned}
& \left.\left(\frac{\mathrm{d} \lambda}{\mathrm{d} \tau}\right)^{-1}\right|_{\lambda=i \omega}=\frac{\Omega}{-\omega^{2} x_{0}+i \omega x_{0} \mathscr{M}} \\
= & \left\{\frac{2 \omega^{2}}{\Lambda}\left[x_{0} \mathscr{M} \cos \omega \tau+x_{0} \omega \sin \omega \tau\right]-\frac{\omega \mathscr{M}}{\Lambda}\left[\omega x_{0} \cos \omega \tau-x_{0} \mathscr{M} \sin \omega \tau\right]-\frac{\omega^{2} x_{0}^{2}}{\Lambda}\right\} \\
& +i\left\{-\frac{2 \omega^{2}}{\Lambda}\left[\omega x_{0} \cos \omega \tau-x_{0} \mathscr{M} \sin \omega \tau\right]-\frac{\omega \mathscr{M}}{\Lambda}\left[x_{0} \mathscr{M} \cos \omega \tau+x_{0} \omega \sin \omega \tau\right]\right. \\
& \left.+\frac{\omega \tau x_{0}^{2} \mathscr{M}^{2}}{\Lambda}+\frac{\omega^{3} \tau x_{0}^{2}}{\Lambda}-\frac{\omega x_{0}^{2} \mathscr{M}}{\Lambda}\right\}
\end{aligned}
$$

where

$$
\begin{aligned}
& \Omega=2 i \omega(\cos \omega \tau+i \sin \omega \tau)+\mathscr{M}(\cos \omega \tau+i \sin \omega \tau)+x_{0}-i \omega \tau x_{0}-\tau x_{0} \mathscr{M}, \\
& \Lambda=\omega^{4} x_{0}^{2}+\omega^{2} x_{0}^{2} \mathscr{M}^{2} .
\end{aligned}
$$

It follows from Eqs. (2.8),(2.9) and (2.15) that

$$
\begin{aligned}
\left.\operatorname{Re}\left(\frac{\mathrm{d} \lambda}{\mathrm{d} \tau}\right)^{-1}\right|_{\lambda=i \omega} & =\frac{2 \omega^{2}}{\Lambda}\left(\omega^{2}-\frac{k y_{0}^{2}}{x_{0}}\right)+\frac{\omega^{2} \mathscr{M}^{2}}{\Lambda}-\frac{\omega^{2} x_{0}^{2}}{\Lambda} \\
& =\frac{2 \omega^{2}-x_{0}^{2}-2 k y_{0}^{2} / x_{0}+\mathscr{M}^{2}}{\omega^{2} x_{0}^{2}+x_{0}^{2} \mathscr{M}^{2}} .
\end{aligned}
$$

From Eq. (2.16), we have

$$
\operatorname{sign}\left\{\operatorname{Re}\left(\frac{\mathrm{d} \lambda}{\mathrm{d} \tau}\right)\right\}_{\lambda=i \omega}=\operatorname{sign}\left\{\operatorname{Re}\left(\frac{\mathrm{d} \lambda}{\mathrm{d} \tau}\right)^{-1}\right\}_{\lambda=i \omega}=\operatorname{sign}\left\{2 \omega^{2}-x_{0}^{2}-\frac{2 k y_{0}^{2}}{x_{0}}+\mathscr{M}^{2}\right\} .
$$

Thus the transversality conditions

$$
\operatorname{sign}\left\{\operatorname{Re}\left(\frac{\mathrm{d} \lambda}{\mathrm{d} \tau}\right)\right\}_{\tau=\tau_{k}^{+}, \omega=\omega_{+}}>0 \text { and } \operatorname{sign}\left\{\operatorname{Re}\left(\frac{\mathrm{d} \lambda}{\mathrm{d} \tau}\right)\right\}_{\tau=\tau_{k}^{-}, \omega=\omega_{-}}<0
$$

are satisfied.

Summarising the considerations above and recalling the results of $[9,17]$, we arrive at the following theorem.

Theorem 2.1. Assume that $\tau>0$ and

$$
\begin{aligned}
& x_{0}+\frac{k y_{0}}{x_{0}}>\frac{m y_{0} E_{0}}{\left(1+m y_{0}\right)^{2}}+\frac{p y_{0} E_{0}}{\left(1+m y_{0}\right)^{2}\left[(p-c m) y_{0}-c\right]}, \\
& k+\frac{k y_{0}}{x_{0}}>\frac{m x_{0} E_{0}}{\left(1+m y_{0}\right)^{2}}+\frac{p x_{0} E_{0}}{\left(1+m y_{0}\right)^{2}\left[(p-c m) y_{0}-c\right]} .
\end{aligned}
$$


Then:

(i) If

$$
\mathscr{M}^{2}>x_{0}^{2}+\frac{2 k y_{0}^{2}}{x_{0}},
$$

then the roots of (2.6) have negative real parts and the interior equilibrium point $X_{0}$ is locally asymptotically stable.

(ii) If $\widehat{\Delta}>0$ and the conditions (2.12),(2.13) holds, then there is a positive integer $N$ such that for

$$
\tau \in\left[0, \tau_{0}^{+}\right) \bigcup\left(\bigcup_{n=0}^{N-1}\left(\tau_{n}^{-}, \tau_{n+1}^{+}\right)\right)
$$

the equilibrium point $X_{0}$ is locally asymptotically stable and it is unstable for

$$
\tau \in\left(\bigcup_{n=0}^{N-1}\left(\tau_{n}^{+}, \tau_{n}^{-}\right)\right) \bigcup\left(\tau_{N}^{+},+\infty\right)
$$

Accordingly, if $\tau$ takes the critical values $\tau_{n}^{ \pm}, n=0,1,2, \ldots, N$, the model (1.5) has Hopf bifurcations.

\section{Stability and Direction of Hopf bifurcations}

We now consider the direction, stability and the period of the Hopf bifurcations. The Hopf bifurcations are usually studied by the center manifold theorem [18]. In particular, the leading order terms in the center manifold have to be determined. For this, we calculate the normal form of system (1.5), starting with the second order Taylor expansions of the locally equivalent parameterised system (2.4) - cf. Appendix B. Thus we have

$$
\begin{aligned}
\dot{y}_{1}(t)= & -x_{0} y_{2}(t)-x_{0} y_{1}(t-\tau)-y_{1}^{2}(t-\tau)-y_{1}(t) y_{2}(t)+\mathscr{O}\left(|Y|^{3}\right), \\
\dot{y}_{2}(t)= & \frac{k y_{0}^{2}}{x_{0}^{2}} y_{1}(t)-\mathscr{M} y_{2}(t)-\frac{k y_{0}^{2}}{x_{0}^{3}} y_{1}^{2}(t)+\frac{2 k y_{0}}{x_{0}^{2}} y_{1}(t) y_{2}(t) \\
& +\left(\frac{m E_{0}}{\left(1+m y_{0}\right)^{3}}+\frac{p E_{0}}{P(m, c)}-\frac{p m y_{0} E_{0}}{P(m, c)}-\frac{p^{2} y_{0} E_{0}}{Q(m, c)}-\frac{k}{x_{0}}\right) y_{2}^{2}(t)+\mathscr{O}\left(|Y|^{3}\right),
\end{aligned}
$$

where

$$
\begin{aligned}
& P(m, c)=\left(1+m y_{0}\right)^{3}\left[(p-c m) y_{0}-c\right], \\
& Q(m, c)=\left(1+m y_{0}\right)^{3}\left[(p-c m) y_{0}-c\right]^{2} .
\end{aligned}
$$

In this section we use the notation of [18] and suppose that for $\tau=\tau_{n}$, the sufficient conditions for the Hopf bifurcation in predator-prey model (1.5) are satisfied. Let $i \omega$ be the corresponding purely imaginary root of (2.6) and

$$
y_{1}(t):=x(\tau t)-x_{0}, \quad y_{2}(t)=y(\tau t)-y_{0}, \quad \tau:=\mu+\tau_{n} .
$$


The Taylor expansions (3.1) can be written as a functional differential equation in $C\left([-1,0], \mathbb{R}^{2}\right)$, viz.

$$
\dot{Y}(t)=L_{\mu}\left(Y_{t}\right)+F\left(\mu, Y_{t}\right)
$$

where

$$
Y(t):=\left(y_{1}(t), y_{2}(t)\right)^{T}, \quad Y_{t}:=Y(t+\theta)=\left(y_{1}(t+\theta), y_{2}(t+\theta)\right), \quad \theta \in[-1,0]
$$

For

$$
\Phi(\theta):=\left(\Phi_{1}(\theta), \Phi_{2}(\theta)\right) \in C\left([-1,0], \mathbb{R}^{2}\right)
$$

we consider the terms

$$
\begin{aligned}
& L_{\mu} \Phi=\left(\tau_{n}+\mu\right)\left(\begin{array}{cc}
0 & -x_{0} \\
\frac{k y_{0}^{2}}{x_{0}^{2}} & -\mathscr{M}
\end{array}\right) \Phi^{T}(0)+\left(\tau_{n}+\mu\right)\left(\begin{array}{cc}
-x_{0} & 0 \\
0 & 0
\end{array}\right) \Phi^{T}(-1), \\
& F(\mu, \Phi(\theta))=\left(\tau_{n}+\mu\right)\left(\begin{array}{l}
F_{11} \\
F_{22}
\end{array}\right),
\end{aligned}
$$

where

$$
\begin{aligned}
F_{11}= & -\Phi_{1}^{2}(-1)-\Phi_{1}(0) \Phi_{2}(0)+\cdots \\
F_{22}= & -\frac{k y_{0}^{2}}{x_{0}^{3}} \Phi_{1}^{2}(0)+\frac{2 k y_{0}}{x_{0}^{2}} \Phi_{1}(0) \Phi_{2}(0) \\
& +\left[\frac{m E_{0}}{\left(1+m y_{0}\right)^{3}}+\frac{p E_{0}}{P(m, c)}-\frac{k}{x_{0}}-\frac{p m y_{0} E_{0}}{P(m, c)}-\frac{p^{2} y_{0} E_{0}}{Q(m, c)}\right] \Phi_{2}^{2}(0)+\cdots
\end{aligned}
$$

By the Riesz representation theorem [17], there exists a $2 \times 2$ matrix function, $\eta(\theta, \mu)$, $-1 \leq \theta \leq 0$ of a bounded variation such that

$$
L_{\mu} \Phi=\int_{-1}^{0}[\mathrm{~d} \eta(\theta, \mu)] \Phi(\theta) \text { for } \Phi(\theta) \in C\left([-1,0], \mathbb{R}^{2}\right) .
$$

Indeed, we choose

$$
\eta(\theta, \mu)=\left(\tau_{n}+\mu\right)\left(\begin{array}{cc}
0 & -x_{0} \\
\frac{k y_{0}^{2}}{x_{0}^{2}} & -\mathscr{M}
\end{array}\right) \delta(\theta)+\left(\tau_{n}+\mu\right)\left(\begin{array}{cc}
-x_{0} & 0 \\
0 & 0
\end{array}\right) \delta(\theta+1),
$$

where

$$
\delta(\theta)= \begin{cases}0, & \theta \neq 0 \\ 1, & \theta=0\end{cases}
$$


and define the operators $A(\mu)$ and $R(\mu)$ acting on functions $\Phi(\theta) \in C:=C^{1}\left([-1,0], \mathbb{R}^{2}\right)$ as

$$
\begin{aligned}
& A(\mu) \Phi(\theta):=\left\{\begin{array}{l}
\frac{\mathrm{d} \Phi(\theta)}{\mathrm{d} \theta}, \quad-1 \leq \theta<0, \\
\int_{-1}^{0} \mathrm{~d} \eta(\theta, \mu) \Phi(\theta), \quad \theta=0
\end{array}\right. \\
& R(\mu) \Phi(\theta):= \begin{cases}0, & -1 \leq \theta<0, \\
F(\mu, \Phi(\theta)), & \theta=0 .\end{cases}
\end{aligned}
$$

The system (3.2) is equivalent to the operator equation

$$
\dot{Y}(t)=A(\mu) Y_{t}+R(\mu) Y_{t},
$$

where $Y_{t}=Y(t+\theta)$ for $\theta \in[-1,0]$.

For $\Psi(s) \in C^{*}:=C^{1}\left([0,1],\left(\mathbb{R}^{2}\right)^{*}\right)$ we define

$$
A^{*} \Psi(s):=\left\{\begin{array}{l}
-\frac{\mathrm{d} \Psi(s)}{\mathrm{d} s}, \quad 0<s \leq 1, \\
\int_{-1}^{0} \mathrm{~d} \eta^{T}(s, 0) \Psi(-s), \quad s=0,
\end{array}\right.
$$

and the bilinear form

$$
<\Psi(s), \Phi(\theta)>:=\bar{\Psi}(0) \Phi(0)-\int_{\theta=-1}^{0} \int_{\xi=0}^{\theta} \bar{\Psi}(\xi-\theta) \mathrm{d} \eta(\theta) \Phi(\xi) \mathrm{d} \xi,
$$

where $\Psi(s) \in C^{1}\left([0,1],\left(\mathbb{R}^{2}\right)^{*}\right),\left(\mathbb{R}^{2}\right)^{*}$ is the 2-dimensional real vector space of row vectors $\Phi(\theta) \in C^{1}\left([-1,0], \mathbb{R}^{2}\right), \eta(\theta)=\eta(\theta, 0)$. Recall that $A(0)$ and $A^{*}$ are mutually adjoint operators [18]. The previous analysis shows that $\pm i \omega \tau_{n}$ are the eigenvalues of the operator $A(0)$. Hence, they are also the eigenvalues of $A^{*}$. Consider the eigenvectors

$$
\begin{aligned}
& q(\theta)=\left(1, q_{2}\right)^{T} e^{i \omega \tau_{n} \theta}, \quad \theta \in[-1,0], \\
& q^{*}(s)=\frac{1}{D}\left(1, q_{2}^{*}\right) e^{i \omega \tau_{n} s}, \quad s \in[0,1]
\end{aligned}
$$

of the operators $A(0)$ and $A^{*}$ associated with the eigenvalues $i \omega \tau_{n}$ and $-i \omega \tau_{n}$, respectively. One can show that

$$
q_{2}=-\frac{i \omega}{x_{0}}-e^{-i \omega \tau_{n}}, \quad q_{2}^{*}=\frac{i \omega}{x_{0}}-e^{-i \omega \tau_{n}}
$$

In addition, we can choose

$$
\bar{D}=1+q_{2} \bar{q}_{2}^{*}-x_{0} \tau_{n} e^{-i \omega \tau_{n}}
$$


such that

$$
<q^{*}(s), q(\theta)>=1 \quad \text { and } \quad<q^{*}(s), \bar{q}(\theta)>=0 .
$$

Thus for the bilinear inner product (3.5), one has

$$
\begin{aligned}
& <q^{*}(s), q(\theta)>=\bar{q}^{*}(0) q(0)-\int_{\theta=-1}^{0} \int_{\xi=0}^{\theta} \overline{q^{*}}(\xi-\theta) \mathrm{d} \eta(\theta) q(\xi) \mathrm{d} \xi \\
& =\frac{1}{\bar{D}}\left\{1+q_{2} \bar{q}_{2}^{*}-\int_{\theta=-1}^{0}\left(1, \bar{q}_{2}^{*}\right) e^{i \omega \tau_{n} \theta} \theta \mathrm{d} \eta(\theta)\left(1, q_{2}\right)^{T}\right\} \\
& =\frac{1}{\bar{D}}\left(1+q_{2} \bar{q}_{2}^{*}-x_{0} \tau_{n} e^{-i \omega \tau_{n}}\right)=1 .
\end{aligned}
$$

It can be shown that $q(\theta)$ and $\bar{q}(\theta)$ are, respectively, the eigenvectors of the operator $A(0)$ corresponding to the eigenvalues $i \omega \tau_{n}$ and $-i \omega \tau_{n}$, i.e.

$$
A(0) \bar{q}(\theta)=-i \omega \tau_{n} \bar{q}(\theta) \text { and } A^{*} q^{*}(s)=-i \omega \tau_{n} q^{*}(s) .
$$

Besides, since $A(0)$ and $A^{*}$ are mutually adjoint operators, the equation

$$
<A^{*} \Psi(s), \Phi(\theta)>=<\Psi(s), A(0) \Phi(\theta)>
$$

holds for any

$$
\Psi(s) \in C^{1}\left([0,1],\left(\mathbb{R}^{2}\right)^{*}\right) \quad \text { and } \quad \Phi(\theta) \in C^{1}\left([-1,0], \mathbb{R}^{2}\right)
$$

Therefore

$$
\begin{aligned}
& <q^{*}(s), \bar{q}(\theta)>=\frac{1}{i \omega \tau_{n}}<-i \omega \tau_{n} q^{*}(s), \bar{q}(\theta)>=\frac{1}{i \omega \tau_{n}}<A^{*} q^{*}(s), \bar{q}(\theta)> \\
& =\frac{1}{i \omega \tau_{n}}<q^{*}(s), A(0) \bar{q}(\theta)>=\frac{1}{i \omega \tau_{n}}<q^{*}(s), \quad-i \omega \tau_{n} \bar{q}(\theta)>, \\
& =-<q^{*}(s), \bar{q}(\theta)>,
\end{aligned}
$$

so that $\left\langle q^{*}(s), \bar{q}(\theta)>=0\right.$.

Following the approach of [18], we determine the coordinates of the center manifold $C_{0}$ at $\mu=0$. Introducing the functions

$$
z(t):=<q^{*}, Y_{t}>\quad \text { and } \quad W(t, \theta):=Y_{t}-2 \operatorname{Re}\{z(t) q(\theta)\}
$$

we write

$$
W(t, \theta)=W(z(t), \bar{z}(t), \theta)=W_{20}(\theta) \frac{z^{2}}{2}+W_{11}(\theta) z \bar{z}+W_{02}(\theta) \frac{\bar{z}^{2}}{2}+W_{30}(\theta) \frac{z^{3}}{6}+\cdots,
$$

where $z$ and $\bar{z}$ are the local coordinates of the center manifold $C_{0}$ in the directions $q$ and $\bar{q}^{*}$. We note that $W$ is real if $Y_{t}$ is real. If $\mu=0$, then for any real solution $Y_{t} \in C_{0}$, the Eq. (3.4) takes the form

$$
\dot{z}(t)=i \omega \tau_{n} z(t)+\bar{q}^{*}(0) F_{0}(z, \bar{z}):=i \omega \tau_{n} z(t)+g(z, \bar{z}),
$$


where

$$
g(z, \bar{z})=g_{20}(\theta) \frac{z^{2}}{2}+g_{11}(\theta) z \bar{z}+g_{02}(\theta) \frac{\bar{z}^{2}}{2}+g_{21}(\theta) \frac{z^{2} \bar{z}}{2}+\cdots .
$$

It follows from the Eq. (3.7) that

$$
g(z, \bar{z})=\bar{q}^{*}(0) F_{0}(z, \bar{z})=\frac{\tau_{n}}{\bar{D}}\left(1, \bar{q}_{2}^{*}\right)\left(\begin{array}{c}
F_{11}^{0} \\
F_{22}^{0}
\end{array}\right)=\frac{\tau_{n}}{\bar{D}}\left(F_{11}^{0}+\bar{q}_{2}^{*} F_{22}^{0}\right),
$$

where

$$
\begin{aligned}
F_{11}^{0}= & -y_{1 t}^{2}(-1)-y_{1 t}(0) y_{2 t}(0)+\cdots \\
F_{22}^{0}= & -\frac{k y_{0}^{2}}{x_{0}^{3}} y_{1 t}^{2}(0)+\frac{2 k y_{0}}{x_{0}^{2}} y_{1 t}(0) y_{2 t}(0) \\
& +\left[\frac{m E_{0}}{\left(1+m y_{0}\right)^{3}}+\frac{p E_{0}}{P(m, c)}-\frac{k}{x_{0}}-\frac{p m y_{0} E_{0}}{P(m, c)}-\frac{p^{2} y_{0} E_{0}}{Q(m, c)}\right] y_{2 t}^{2}(0)+\cdots .
\end{aligned}
$$

Using (3.6)-(3.8), we represent $g(z, \bar{z})$ in the form

$$
\begin{aligned}
& g(z, \bar{z})=\frac{\tau_{n}}{\bar{D}}\left\{z ^ { 2 } \left[-e^{-2 i \omega \tau_{n} \theta}-q_{2}-\frac{k \bar{q}_{2}^{*} y_{0}^{2}}{x_{0}^{3}}+\frac{2 k q_{2} \bar{q}_{2}^{*} y_{0}}{x_{0}^{2}}+\frac{m q_{2}^{2} \bar{q}_{2}^{*} E_{0}}{\left(1+m y_{0}\right)^{3}}\right.\right. \\
& \left.+\frac{p q_{2}^{2} \overline{q_{2}^{*}} E_{0}}{P(m, c)}-\frac{p m q_{2}^{2} \bar{q}_{2}^{*} y_{0} E_{0}}{P(m, c)}-\frac{p^{2} q_{2}^{2} \overline{q_{2}^{*} y_{0} E_{0}}}{Q(m, c)}-\frac{k q_{2}^{2} \overline{q_{2}^{*}}}{x_{0}}\right] \\
& +z \bar{z}\left[-2-2 \operatorname{Re}\left(q_{2}\right)-\frac{2 k \overline{q_{2}^{*}} y_{0}^{2}}{x_{0}^{3}}+\frac{4 k \overline{q_{2}^{*}} y_{0}}{x_{0}^{2}} \operatorname{Re}\left(q_{2}\right)+\frac{2 m q_{2} \overline{q_{2}} \overline{q_{2}^{*}} E_{0}}{\left(1+m y_{0}\right)^{3}}\right. \\
& \left.+\frac{2 p q_{2} \overline{q_{2}} \overline{q_{2}^{*}} E_{0}}{P(m, c)}-\frac{2 p m q_{2} \overline{q_{2}} \overline{q_{2}^{*}} y_{0} E_{0}}{P(m, c)}-\frac{2 p^{2} q_{2} \overline{q_{2}} \overline{q_{2}^{*}} y_{0} E_{0}}{Q(m, c)}-\frac{2 k q_{2} \overline{q_{2}} \overline{q_{2}^{*}}}{x_{0}}\right] \\
& +\bar{z}^{2}\left[-e^{2 i \omega \tau_{n} \theta}-\overline{q_{2}}-\frac{k \overline{q_{2}^{*} y_{0}^{2}}}{x_{0}^{3}}+\frac{2 k \overline{q_{2}} \overline{q_{2}^{*}} y_{0}}{x_{0}^{2}}+\frac{m{\overline{q_{2}}}^{2} \overline{q_{2}^{*}} E_{0}}{\left(1+m y_{0}\right)^{3}}\right. \\
& \left.+\frac{p{\overline{q_{2}}}^{2}{\overline{q_{2}^{*}}}^{*} E_{0}}{P(m, c)}-\frac{p m{\overline{q_{2}}}^{2} \overline{q_{2}^{*} y_{0} E_{0}}}{P(m, c)}-\frac{p^{2} \overline{q_{2}}{ }^{2} \overline{q_{2}^{*} y_{0} E_{0}}}{Q(m, c)}-\frac{k{\overline{q_{2}}}^{2} \overline{q_{2}^{*}}}{x_{0}}\right] \\
& +z^{2} \bar{z}\left[\left(-q_{2}-\frac{2 k q_{2}^{*} y_{0}^{2}}{x_{0}^{3}}+\frac{2 k q_{2} \bar{q}_{2}^{*} y_{0}}{x_{0}^{2}}\right) W_{11}^{(1)}(0)\right. \\
& +\left(-1+\frac{2 k q_{2}^{*} y_{0}}{x_{0}^{2}}+\frac{2 m q_{2} \bar{q}_{2}^{*} E_{0}}{\left(1+m y_{0}\right)^{3}}+\frac{2 p q_{2} \bar{q}_{2}^{*} E_{0}}{P(m, c)}-\frac{2 k q_{2} \bar{q}_{2}^{*}}{x_{0}}-\frac{2 p m q_{2} \bar{q}_{2}^{*} y_{0} E_{0}}{P(m, c)}\right. \\
& \left.-\frac{2 p^{2} q_{2} \overline{q_{2}^{*} y_{0} E_{0}}}{Q(m, c)}\right) W_{11}^{(2)}(0)+\left(-\frac{\overline{q_{2}}}{2}-\frac{k q_{2}^{*} y_{0}^{2}}{x_{0}^{3}}+\frac{k \overline{q_{2}} \overline{q_{2}^{*}} y_{0}}{x_{0}^{2}}\right) W_{20}^{(1)}(0)
\end{aligned}
$$




$$
\begin{aligned}
& +\left(-\frac{1}{2}+\frac{k \overline{q_{2}^{*} y_{0}}}{x_{0}^{2}}+\frac{m \overline{q_{2}} \overline{q_{2}^{*}} E_{0}}{\left(1+m y_{0}\right)^{3}}+\frac{p \overline{q_{2}} \overline{q_{2}^{*}} E_{0}}{P(m, c)}\right. \\
& \left.--\frac{p m \overline{q_{2}} \overline{q_{2}^{*}} y_{0} E_{0}}{P(m, c)} \frac{p^{2} \overline{q_{2}} \overline{q_{2}^{*}} y_{0} E_{0}}{Q(m, c)}-\frac{k \overline{q_{2}} \overline{q_{2}^{*}}}{x_{0}}\right) W_{20}^{(2)}(0) \\
& \left.\left.-2 e^{-i \omega \tau_{n} \theta} W_{11}^{(1)}(-1)-e^{i \omega \tau_{n} \theta} W_{20}^{(1)}(-1)\right]+\cdots\right\} .
\end{aligned}
$$

Equating the coefficients (3.8) and (3.9) shows that

$$
\begin{aligned}
& g_{20}=\frac{2 \tau_{n}}{\bar{D}}\left[-e^{-2 i \omega \tau_{n} \theta}-q_{2}-\frac{k \bar{q}_{2}^{*} y_{0}^{2}}{x_{0}^{3}}+\frac{2 k q_{2} \bar{q}_{2}^{*} y_{0}}{x_{0}^{2}}+\frac{m q_{2}^{2} \bar{q}_{2}^{*} E_{0}}{\left(1+m y_{0}\right)^{3}}\right. \\
& \left.+\frac{p q_{2}^{2} q_{2}^{*} E_{0}}{P(m, c)}-\frac{p m q_{2}^{2} \bar{q}_{2}^{*} y_{0} E_{0}}{P(m, c)}-\frac{p^{2} q_{2}^{2} \bar{q}_{2}^{*} y_{0} E_{0}}{Q(m, c)}-\frac{k q_{2}^{2} \bar{q}_{2}^{*}}{x_{0}}\right], \\
& g_{11}=\frac{\tau_{n}}{\bar{D}}\left[-2-2 \operatorname{Re}\left(q_{2}\right)-\frac{2 k \overline{q_{2}^{*}} y_{0}^{2}}{x_{0}^{3}}+\frac{4 k \overline{q_{2}^{*}} y_{0}}{x_{0}^{2}} \operatorname{Re}\left(q_{2}\right)+\frac{2 m q_{2} \overline{q_{2}} \overline{q_{2}^{*}} E_{0}}{\left(1+m y_{0}\right)^{3}}\right. \\
& \left.+\frac{2 p q_{2} \overline{q_{2}} \overline{q_{2}^{*}} E_{0}}{P(m, c)}-\frac{2 p m q_{2} \overline{q_{2}} \overline{q_{2}^{*} y_{0} E_{0}}}{P(m, c)}-\frac{2 p^{2} q_{2} \overline{q_{2}} \overline{q_{2}^{*}} y_{0} E_{0}}{Q(m, c)}-\frac{2 k q_{2} \overline{q_{2}} \overline{q_{2}^{*}}}{x_{0}}\right] \text {, }
\end{aligned}
$$

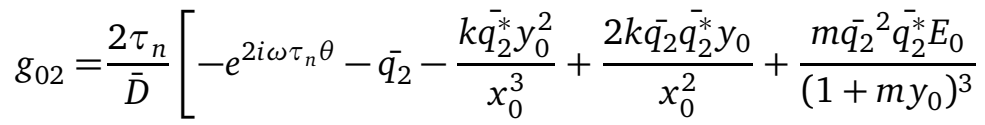

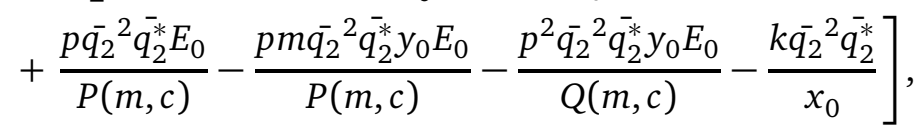

$$
\begin{aligned}
& g_{21}=\frac{2 \tau_{n}}{\bar{D}}\left[\left(-q_{2}-\frac{2 k q_{2}^{*} y_{0}^{2}}{x_{0}^{3}}+\frac{2 k q_{2} \bar{q}_{2}^{*} y_{0}}{x_{0}^{2}}\right) W_{11}^{(1)}(0)\right. \\
& +\left(-1+\frac{2 k q_{2}^{*} y_{0}}{x_{0}^{2}}+\frac{2 m q_{2} q_{2}^{*} E_{0}}{\left(1+m y_{0}\right)^{3}}+\frac{2 p q_{2} \bar{q}_{2}^{*} E_{0}}{P(m, c)}-\frac{2 k q_{2} q_{2}^{*}}{x_{0}}\right. \\
& \left.-\frac{2 p m q_{2} \bar{q}_{2}^{*} y_{0} E_{0}}{P(m, c)}-\frac{2 p^{2} q_{2} \bar{q}_{2}^{*} y_{0} E_{0}}{Q(m, c)}\right) W_{11}^{(2)}(0) \\
& +\left(-\frac{\overline{q_{2}}}{2}-\frac{k q_{2}^{*} y_{0}^{2}}{x_{0}^{3}}+\frac{k \overline{q_{2}} \overline{q_{2}^{*}} y_{0}}{x_{0}^{2}}\right) W_{20}^{(1)}(0)+\left(-\frac{1}{2}+\frac{k \overline{q_{2}^{*}} y_{0}}{x_{0}^{2}}+\frac{m \overline{q_{2}} \overline{q_{2}^{*}} E_{0}}{\left(1+m y_{0}\right)^{3}}\right. \\
& \left.+\frac{p \overline{q_{2}} \overline{q_{2}^{*}} E_{0}}{P(m, c)}-\frac{p m \overline{q_{2}} \overline{q_{2}^{*}} y_{0} E_{0}}{P(m, c)}-\frac{p^{2} \overline{q_{2}} \overline{q_{2}^{*} y_{0} E_{0}}}{Q(m, c)}-\frac{k \overline{q_{2}} \overline{q_{2}^{*}}}{x_{0}}\right) W_{20}^{(2)}(0) \\
& \left.-2 e^{-i \omega \tau_{n} \theta} W_{11}^{(1)}(-1)-e^{i \omega \tau_{n} \theta} W_{20}^{(1)}(-1)\right] \text {. }
\end{aligned}
$$

The coefficients $g_{20}, g_{11}$ and $g_{02}$ are now expressed via known parameters, but formula for $g_{21}$ contains not yet determined terms $W_{20}(\theta)$ and $W_{11}(\theta)$. Following the considerations 
of [18], we write

$$
\begin{aligned}
& W_{20}(\theta)=\frac{i g_{20}}{\omega \tau_{n}} q(0) e^{i \omega \tau_{n} \theta}+\frac{i \bar{g}_{02}}{3 \omega \tau_{n}} \bar{q}(0) e^{-i \omega \tau_{n} \theta}+\mathscr{E}_{1} e^{2 i \omega \tau_{n} \theta}, \\
& W_{11}(\theta)=-\frac{i g_{11}}{\omega \tau_{n}} q(0) e^{i \omega \tau_{n} \theta}+\frac{i \bar{g}_{11}}{\omega \tau_{n}} \bar{q}(0) e^{-i \omega \tau_{n} \theta}+\mathscr{E}_{2},
\end{aligned}
$$

where

$$
\begin{aligned}
& \mathscr{E}_{1}=2\left(\begin{array}{cc}
2 i \omega+x_{0} e^{-2 i \omega \tau_{n}} & x_{0} \\
-\frac{k y_{0}^{2}}{x_{0}^{2}} & 2 i \omega+\mathscr{M}
\end{array}\right)^{-1}\left(\begin{array}{l}
\mathscr{P}_{11} \\
\mathscr{P}_{21}
\end{array}\right), \\
& \mathscr{E}_{2}=2\left(\begin{array}{cc}
x_{0} & x_{0} \\
-\frac{k y_{0}^{2}}{x_{0}^{2}} & \mathscr{M}
\end{array}\right)^{-1}\left(\begin{array}{l}
\mathscr{Q}_{11} \\
\mathscr{Q}_{21}
\end{array}\right),
\end{aligned}
$$

and

$$
\begin{aligned}
\mathscr{P}_{11}= & -e^{-2 i \omega \tau_{n}}-q_{2}, \\
\mathscr{P}_{21}= & -\frac{k y_{0}^{2}}{x_{0}^{3}}+\frac{2 k q_{2} y_{0}}{x_{0}^{2}}+\frac{m q_{2}^{2} E_{0}}{\left(1+m y_{0}\right)^{3}}+\frac{p q_{2}^{2} E_{0}}{P(m, c)}-\frac{p m q_{2}^{2} y_{0} E_{0}}{P(m, c)} \\
& -\frac{p^{2} q_{2}^{2} y_{0} E_{0}}{Q(m, c)}-\frac{k q_{2}^{2}}{x_{0}}, \\
\mathscr{Q}_{11}= & -1-\operatorname{Re}\left(q_{2}\right), \\
\mathscr{Q}_{21}= & -\frac{k y_{0}^{2}}{x_{0}^{3}}+\frac{2 k y_{0}}{x_{0}^{2}} \operatorname{Re}\left(q_{2}\right)+\frac{m q_{2} \overline{q_{2}} E_{0}}{\left(1+m y_{0}\right)^{3}}+\frac{p q_{2} \overline{q_{2}} E_{0}-p m q_{2} \overline{q_{2} y_{0} E_{0}}}{P(m, c)} \\
& -\frac{p^{2} q_{2} \overline{q_{2}} y_{0} E_{0}}{Q(m, c)}-\frac{k q_{2} \overline{q_{2}}}{x_{0}} .
\end{aligned}
$$

Straightforward calculations show that

$$
\begin{aligned}
& \mathscr{E}_{1}=\left(\begin{array}{c}
\frac{4 \mathscr{P}_{11} i \omega+2 \mathscr{M} \mathscr{P}_{11}-2 x_{0} \mathscr{P}_{21}}{\varpi} \\
\frac{4 i \omega \mathscr{P}_{21}+2 x_{0} e^{-2 i \omega \tau_{n} \mathscr{P}_{21}+\left(2 k y_{0}^{2} / x_{0}^{2}\right) \mathscr{P}_{11}}}{\varpi}
\end{array}\right)_{2 \times 1}, \\
& \mathscr{E}_{2}=\left(\begin{array}{c}
\frac{2 \mathscr{M} \mathscr{Q}_{11}-2 x_{0} \mathscr{Q}_{21}}{k y_{0}^{2} / x_{0}+x_{0} \mathscr{M}} \\
\frac{\left(2 k y_{0}^{2} / x_{0}^{2}\right) \mathscr{Q}_{11}+2 x_{0} \mathscr{Q}_{21}}{k y_{0}^{2} / x_{0}+x_{0} \mathscr{M}}
\end{array}\right)_{2 \times 1},
\end{aligned}
$$


where

$$
\varpi=\frac{k y_{0}^{2}}{x_{0}}+x_{0} \mathscr{M} e^{-2 i \omega \tau_{n}}+2 i \omega\left(\mathscr{M}+x_{0} e^{-2 i \omega \tau_{n}}\right)-4 \omega^{2} .
$$

Substituting $\mathscr{E}_{1}, \mathscr{E}_{2}$ into (3.11), we get the expressions for $W_{20}(\theta)$ and $W_{11}(\theta)$. This leads to complete description of $g_{21}$ in (3.10) and we can compute important characteristic of [18], viz.

$$
\begin{aligned}
& c_{1}(0):=\frac{i}{2 \omega \tau_{n}}\left(g_{11} g_{20}-2\left|g_{11}\right|^{2}-\frac{\left|g_{02}\right|^{2}}{3}\right)+\frac{g_{21}}{2}, \\
& \mu_{2}:=-\frac{\operatorname{Re}\left\{c_{1}(0)\right\}}{\operatorname{Re}\left\{\lambda^{\prime}\left(\tau_{n}\right)\right\}}, \quad \beta_{2}:=2 \operatorname{Re}\left\{c_{1}(0)\right\}, \\
& T_{2}:=-\frac{\operatorname{Im}\left\{c_{1}(0)\right\}+\mu_{2} \operatorname{Im}\left\{\lambda^{\prime}\left(\tau_{n}\right)\right\}}{\omega \tau_{n}},
\end{aligned}
$$

where the sign of $\mu_{2}$ determines the direction of the Hopf bifurcation on the center manifold $C_{0}$ for $\tau=\tau_{n}$, the sign of $\beta_{2}$ the stability of the bifurcating periodic orbits, and the sign of $T_{2}$ the period of the bifurcating periodic orbits. Recalling the conditions for the Hopf bifurcations - cf. Section 2 and the Hopf bifurcation theorem in [18], we arrive at the following result.

Theorem 3.1. Assume that $\tau>0, \widehat{\Delta}>0$,

$$
\begin{aligned}
& x_{0}+\frac{k y_{0}}{x_{0}}>\frac{m y_{0} E_{0}}{\left(1+m y_{0}\right)^{2}}+\frac{p y_{0} E_{0}}{\left(1+m y_{0}\right)^{2}\left[(p-c m) y_{0}-c\right]}, \\
& k+\frac{k y_{0}}{x_{0}}>\frac{m x_{0} E_{0}}{\left(1+m y_{0}\right)^{2}}+\frac{p x_{0} E_{0}}{\left(1+m y_{0}\right)^{2}\left[(p-c m) y_{0}-c\right]},
\end{aligned}
$$

and the conditions (2.12),(2.13) hold. If $\tau$ takes the critical values $\tau_{n}^{ \pm}, n=0,1,2, \ldots, N$, then the model (1.5) has Hopf bifurcations. Moreover,

(i) If $\mu_{2}>0\left(\mu_{2}<0\right)$, then the Hopf bifurcations are supercritical (subcritical).

(ii) If $\beta_{2}<0\left(\beta_{2}>0\right)$, then the bifurcating periodic orbits are stable (unstable).

(iii) If $T_{2}>0\left(T_{2}<0\right)$, then the bifurcating periodic orbits increase (decrease).

\section{Singularity Induced Bifurcation and Feedback Control}

We now show that for time delay $\tau=0$ the model (1.5) can have a singularity-induced bifurcation. According to $[8,14]$, if the harvesting profit $v$ is equal to zero, then there exists a biological economic equilibrium. Therefore, we choose $v$ as a the bifurcation parameter to study the singularity-induced bifurcation around $v_{0}=0$.

It is easily seen that the differential-algebra system (1.5) without delay has an equilibrium point

$$
\left(X_{*}, v_{0}\right)=\left(x_{*}, y_{*}, E_{*}, v_{0}\right)=\left(\frac{[a p-c(a m+1)]}{(p-c m)}, \frac{c}{(p-c m)},\left(1+m y_{*}\right)\left(d-\frac{k y_{*}}{x_{*}}\right), v_{0}\right) .
$$


Moreover, some ecological reasons require the positivity of $x_{*}, y_{*}$ and $E_{*}$, hence we assume that

$$
p>c m, \quad a p>c(a m+1), \quad d>\frac{k y_{*}}{x_{*}} .
$$

The singularity-induced bifurcation theorem in Ref. [35] — cf. Appendix C, yields the following result.

Theorem 4.1. If the model (1.5) without delay has a singularity-induced bifurcation, then the bifurcation value is $v_{0}=0$. Moreover, the equilibrium point $X_{*}$ changes from stable to unstable when the harvesting profit $v$ increases through zero.

Proof. Setting

$$
\Upsilon:=\left(\begin{array}{lll}
1 & 0 & 0 \\
0 & 1 & 0 \\
0 & 0 & 0
\end{array}\right), \quad \bar{f}(X(t), v):=\left(\begin{array}{c}
\bar{f}_{1}(X(t), v) \\
\bar{f}_{2}(X(t), v) \\
\bar{g}(X(t), v)
\end{array}\right)=\left(\begin{array}{c}
x(t)(a-x(t)-y(t)) \\
y(t)\left(d-k \frac{y(t)}{x(t)}-\frac{E(t)}{1+m y(t)}\right) \\
E(t)\left(\frac{p y(t)}{1+m y(t)}-c\right)-v
\end{array}\right),
$$

we write the differential-algebra system (1.5) as

$$
\Upsilon \dot{X}(t)=\bar{f}(X(t), v),
$$

where $X(t)=(x(t), y(t), E(t))^{T}$. The leading matrix $\Upsilon$ is singular and in such cases, differential-algebra systems are called singular or degenerate. Next, we check the conditions (SI1-SI3) in [35, Part III(A)] for singularity-induced bifurcation.

(I) One can show that $\operatorname{det}\left(\left.D_{E} \bar{g}(X(t), v)\right|_{\left(X_{*}, v_{0}\right)}\right)=0$, so that $D_{E} \bar{g}(X(t), v)$ has an algebraically simple zero eigenvalue at $\left(X_{*}, v_{0}\right)$. Besides,

$$
\operatorname{trace}\left(\left(\begin{array}{c}
D_{E} \bar{f}_{1} \\
D_{E} \bar{f}_{2}
\end{array}\right) \operatorname{adj}\left(D_{E} \bar{g}\right)\left(D_{x} \bar{g}, D_{y} \bar{g}\right)\right)_{\left(X_{*}, v_{0}\right)}=-\frac{p E_{*} y_{*}}{\left(1+m y_{*}\right)^{3}}<0
$$

(II)

$$
\begin{aligned}
\operatorname{det}\left(J_{1}\right) & :=\operatorname{det}\left(\begin{array}{ccc}
D_{x} \bar{f}_{1} & D_{y} \bar{f}_{1} & D_{E} \bar{f}_{1} \\
D_{x} \bar{f}_{2} & D_{y} \bar{f}_{2} & D_{E} \bar{f}_{2} \\
D_{x} \bar{g} & D_{y} \bar{g} & D_{E} \bar{g}
\end{array}\right)_{\left(X_{*}, v_{0}\right)} \\
& =\operatorname{det}\left(\begin{array}{ccc}
-x_{*} & -x_{*} & 0 \\
\frac{k y_{*}^{2}}{x_{*}^{2}} & -\frac{k y_{*}}{x_{*}}+\frac{m E_{*} y_{*}}{\left(1+m y_{*}\right)^{2}} & -\frac{y_{*}}{1+m y_{*}} \\
0 & \frac{p E_{*}}{\left(1+m y_{*}\right)^{2}} & 0
\end{array}\right)=-\frac{p x_{*} y_{*} E_{*}}{\left(1+m y_{*}\right)^{3}}<0 .
\end{aligned}
$$

Hence, the matrix $J_{1}$ is nonsingular at $\left(X_{*}, v_{0}\right)$. 
(III) Following [35, Part IV], we define the function

$$
\Delta(X(t), v):=\operatorname{det}\left(D_{E} \bar{g}(X(t), v)=\frac{p y}{(1+m y)}-c,\right.
$$

and calculate the determinant $\operatorname{det}\left(J_{2}\right)$, i.e.

$$
\begin{aligned}
& \operatorname{det}\left(\begin{array}{cccc}
D_{x} \bar{f}_{1} & D_{y} \bar{f}_{1} & D_{E} \bar{f}_{1} & D_{v} \bar{f}_{1} \\
D_{x} \bar{f}_{2} & D_{y} \bar{f}_{2} & D_{E} \bar{f}_{2} & D_{v} \bar{f}_{2} \\
D_{x} \bar{g} & D_{y} \bar{g} & D_{E} \bar{g} & D_{v} \bar{g} \\
D_{x} \Delta & D_{y} \Delta & D_{E} \Delta & D_{v} \Delta
\end{array}\right)_{\left(X_{*}, v_{0}\right)} \\
= & \left(\begin{array}{cccc}
-x_{*} & -x_{*} & 0 & 0 \\
\frac{k y_{*}^{2}}{x_{*}^{2}} & -\frac{k y_{*}}{x_{*}}+\frac{m E_{*} y_{*}}{\left(1+m y_{*}\right)^{2}} & -\frac{y_{*}}{1+m y_{*}} & 0 \\
0 & \frac{p E_{*}}{\left(1+m y_{*}\right)^{2}} & 0 & -1 \\
0 & \frac{p}{\left(1+m y_{*}\right)^{2}} & 0 & 0
\end{array}\right)=-\frac{p x_{*} y_{*}}{\left(1+m y_{*}\right)^{3}}<0 .
\end{aligned}
$$

Thus the matrix $J_{2}$ is also nonsingular at $\left(X_{*}, v_{0}\right)$.

The items (I)-(III) show that the conditions of [35, Theorem 3] are satisfied. Consequently, the differential-algebra system (4.2) can have a singularity-induced bifurcation with the bifurcation value $v_{0}=0$.

Further, in order to determine the stability switch of the equilibrium point $X_{*}$ we need to establish the following terms:

$$
\begin{aligned}
& \bar{b}:=-\operatorname{trace}\left(\left(\begin{array}{l}
D_{E} \bar{f}_{1} \\
D_{E} \bar{f}_{2}
\end{array}\right) \operatorname{adj}\left(D_{E} \bar{g}\right)\left(D_{x} \bar{g}, D_{y} \bar{g}\right)\right)_{\left(X_{*}, v_{0}\right)}=\frac{p E_{*} y_{*}}{\left(1+m y_{*}\right)^{3}}>0, \\
& \bar{c}:=\left(D_{v} \Delta-\left(D_{x} \Delta, D_{y} \Delta, D_{E} \Delta\right)\left(\begin{array}{ccc}
D_{x} \bar{f}_{1} & D_{y} \bar{f}_{1} & D_{E} \bar{f}_{1} \\
D_{x} \bar{f}_{2} & D_{y} \bar{f}_{2} & D_{E} \bar{f}_{2} \\
D_{x} \bar{g} & D_{y} \bar{g} & D_{E} \bar{g}
\end{array}\right)^{-1}\left(\begin{array}{c}
D_{v} \bar{f}_{1} \\
D_{v} \bar{f}_{2} \\
D_{v} \bar{g}
\end{array}\right)\right)_{\left(X_{*}, v_{0}\right)} \\
& =\epsilon\left(\begin{array}{ccc}
-\frac{1}{x_{*}} & 0 & -\frac{\left(1+m y_{*}\right)^{2}}{p E_{*}} \\
0 & 0 & \frac{\left(1+m y_{*}\right)^{2}}{p E_{*}} \\
-\frac{k y_{*}\left(1+m y_{*}\right)}{x_{*}^{3}} & -\frac{1+m y_{*}}{y_{*}} & -\frac{\left(1+m y_{*}\right)^{3}}{p x_{*} y_{*} E_{*}}\left[k y_{*}+\frac{k y_{*}^{2}}{x_{*}}-\frac{m x_{*} y_{*} E_{*}}{\left(1+m y_{*}\right)^{2}}\right]
\end{array}\right) \\
& \times\left(\begin{array}{c}
0 \\
0 \\
-1
\end{array}\right)=\frac{1}{E_{*}}>0
\end{aligned}
$$


where

$$
\epsilon:=\left(0,-\frac{p}{\left(1+m y_{*}\right)^{2}}, 0\right)
$$

so that

$$
\frac{b}{\bar{c}}=\frac{p E_{*}^{2} y_{*}}{\left(1+m y_{*}\right)^{3}}>0 .
$$

According [35, Theorem 3], if $v$ increases through $v_{0}$, then one eigenvalue $\lambda_{1}$ of the differential-algebra system (4.2) moves along the real axis from $\mathbb{C}^{-}$to $\mathbb{C}^{+}$diverging to $\infty$ since $\bar{b} / \bar{c}>0$.

On the other hand, the Jacobi matrix of the differential-algebraic system (4.2) at the equilibrium point $X_{*}$ is

$$
J_{X_{*}}=\left(\begin{array}{ccc}
-x_{*} & -x_{*} & 0 \\
\frac{k y_{*}^{2}}{x_{*}^{2}} & -\frac{k y_{*}}{x_{*}}+\frac{m E_{*} y_{*}}{\left(1+m y_{*}\right)^{2}} & -\frac{y_{*}}{1+m y_{*}} \\
0 & \frac{p E_{*}}{\left(1+m y_{*}\right)^{2}} & 0
\end{array}\right) .
$$

Considering now the characteristic equation $\operatorname{det}\left(\lambda \Upsilon-J_{X_{*}}\right)=0$ of the system (4.2), we observe that $\lambda_{2}=-x_{*}$ is also an eigenvalue of the system (4.2) at the equilibrium point $X_{*}$. It is negative and continuous. Therefore, according to [35], it cannot jump from one half open complex plane to another as the harvesting profit $v$ increases through the point $v_{0}=0$. Thus $\lambda_{2}$ is continuous and bounded in the half-plane $\mathbb{C}^{-}$when profit $v$ increases through zero. Therefore, the movement $\lambda_{2}$ does not influence the stability of the positive equilibrium point $X_{*}$ of the system (4.2).

Table 1 shows the change of the sign in the real part of $\lambda_{1}$ and $\lambda_{2}$ caused by harvesting profit $v$.

Table 1: Signs of real parts of eigenvalues of differential-algebraic system (4.2) at $X_{*}$.

\begin{tabular}{||ccc||}
\hline Harvesting profit $v$ & $\operatorname{Re} \lambda_{1}$ & $\operatorname{Re} \lambda_{2}$ \\
\hline$v<0$ & - & - \\
$v>0$ & + & - \\
\hline
\end{tabular}

By the Routh-Hurwitz criteria [34], for negative $v$ the equilibrium point $X_{*}$ of the system (4.2) is stable. It becomes unstable when the harvesting profit $v$ increases through zero. Consequently, there is a stability switch (from stable to unstable) for the equilibrium point $X_{*}$. This completes the proof.

Remark 4.1. The singularity-induced bifurcation is a new type of bifurcation in differentialalgebraic systems discovered by Venkatasubramanian et al. [35]. Later on, important results on singularity-induced bifurcations have been obtained in [2, 3, 38]. It is notable that singularity-induced bifurcations do not appear in the systems described by differential equations. 
According to [35], the singularity-induced bifurcations lead to impulsive phenomenon in the system (4.2) that is harmful for the system. In what follows, we develop a state feedback controller to eliminate the singularity-induced bifurcation at the equilibrium point $X_{*}$. Moreover, by [10, Theorem 2-2.1] the differential-algebraic system (4.2) is locally controllable at the equilibrium point $X_{*}$, since the matrix discriminant matrix $\left(J_{X_{*}}, \Upsilon J_{X_{*}}, \Upsilon^{2} J_{X_{*}}\right)$ at the equilibrium point $X_{*}$ has rank 3. Therefore, according to [10, Theorem 3-1.2], in order to stabilise the system (4.2) at its equilibrium point $X_{*}$, a state feedback controller can be constructed. Following [10], we consider the state feedback controller of the form

$$
\bar{u}=\left(\begin{array}{l}
0 \\
0 \\
1
\end{array}\right)\left(\begin{array}{lll}
0 & 0 & \bar{\sigma}
\end{array}\right)\left(\begin{array}{c}
x(t)-x_{*} \\
y(t)-y_{*} \\
E(t)-E_{*}
\end{array}\right),
$$

where $\bar{\sigma}$ is the feedback gain and $E_{*}$ the third component of the equilibrium point $X_{*}$. Applying the state feedback controller $\bar{u}=\bar{\sigma}\left(E-E_{*}\right)$ to (4.2), we arrive at the controlled differential-algebraic system

$$
\begin{aligned}
& \dot{x}(t)=x(t)(a-x(t)-y(t)) \\
& \dot{y}(t)=y(t)\left(d-k \frac{y(t)}{x(t)}-\frac{E(t)}{1+m y(t)}\right) \\
& 0=E(t)\left(\frac{p y(t)}{1+m y(t)}-c\right)+\bar{\sigma}\left(E-E_{*}\right)-v
\end{aligned}
$$

Theorem 4.2. If the feedback gain $\bar{\sigma}$ satisfies the inequality

$$
\begin{aligned}
\bar{\sigma}>\max \left\{\frac{p x_{*} y_{*} E_{*}}{\left(x_{*}^{2}+k y_{*}\right)\left(1+m y_{*}\right)^{3}-m x_{*} y_{*} E_{*}\left(1+m y_{*}\right)},\right. \\
\left.\frac{p x_{*}^{2} y_{*} E_{*}}{k y_{*}\left(x_{*}+y_{*}\right)\left(1+m y_{*}\right)^{3}-m x_{*}^{2} y_{*} E_{*}\left(1+m y_{*}\right)}\right\},
\end{aligned}
$$

then the controlled differential-algebraic system (4.3) can be stabilised at the equilibrium point $X_{*}$ of the initial system (4.2).

Proof. The Jacobi matrix of the system (4.3) at $\left(X_{*}, v_{0}\right)$ is

$$
\bar{J}_{0}=\left(\begin{array}{ccc}
-x_{*} & -x_{*} & 0 \\
\frac{k y_{*}^{2}}{x_{*}^{2}} & -\frac{k y_{*}}{x_{*}}+\frac{m E_{*} y_{*}}{\left(1+m y_{*}\right)^{2}} & -\frac{y_{*}}{1+m y_{*}} \\
0 & \frac{p E_{*}}{\left(1+m y_{*}\right)^{2}} & \bar{\sigma}
\end{array}\right),
$$

and the corresponding characteristic equation has the form

$$
\lambda^{2}+\bar{a}_{1} \lambda+\bar{a}_{2}=0,
$$


where

$$
\begin{aligned}
& \bar{a}_{1}=x_{*}+\frac{k y_{*}}{x_{*}}-\frac{m E_{*} y_{*}}{\left(1+m y_{*}\right)^{2}}-\frac{p E_{*} y_{*}}{\bar{\sigma}\left(1+m y_{*}\right)^{3}}, \\
& \bar{a}_{2}=k y_{*}+\frac{k y_{*}^{2}}{x_{*}}-\frac{m x_{*} y_{*} E_{*}}{\left(1+m y_{*}\right)^{2}}-\frac{p x_{*} y_{*} E_{*}}{\bar{\sigma}\left(1+m y_{*}\right)^{3}} .
\end{aligned}
$$

We observe that:

(i) If $\bar{a}_{1}>0, \bar{a}_{2}>0$, and $\bar{a}_{1}^{2}>4 \bar{a}_{2}$, then (4.5) has two negative real roots.

(ii) If $\bar{a}_{1}>0, \bar{a}_{2}>0$, and $\bar{a}_{1}^{2}=4 \bar{a}_{2}$, then (4.5) has one negative real root.

(iii) If $\bar{a}_{1}>0, \bar{a}_{2}>0$, and $\bar{a}_{1}^{2}<4 \bar{a}_{2}$, then (4.5) has complex roots with negative real parts.

The equilibrium point $X_{*}$ of the system (4.3) is either a stable node (in cases (i) and (ii)) or a stable focus (in case (iii)) when both of the coefficients $\bar{a}_{1}$ and $\bar{a}_{2}$ are positive cf. Ref. [34]. At this moment, the controlled differential-algebraic system (4.3) is stable. Subsequently, it follows from the inequalities $\bar{a}_{1}>0$ and $\bar{a}_{2}>0$ that the feedback gain $\bar{\sigma}$ satisfies the inequality (4.4). Hence, the initial differential-algebra system (4.2) can be stabilised at the equilibrium point $X_{*}$ by the feedback controller $\bar{u}=\bar{\sigma}\left(E-E_{*}\right)$. This allows to eliminate the singularity-induced bifurcation in (4.2).

\section{Numerical Simulations}

We consider two numerical examples to illustrate the theoretical findings.

Example 5.1 (Hopf Bifurcation). Using the parameters

$$
a=5, \quad d=\frac{7}{4}, \quad k=1, \quad m=1, \quad p=1, \quad c=\frac{1}{2}, \quad v=\frac{1}{4}
$$

we obtain the following problem

$$
\begin{aligned}
& \dot{x}(t)=x(t)(5-x(t-\tau)-y(t)), \\
& \dot{y}(t)=y(t)\left(\frac{7}{4}-\frac{y(t)}{x(t)}-\frac{E(t)}{1+y(t)}\right), \\
& 0=E(t)\left(\frac{y(t)}{1+y(t)}-\frac{1}{2}\right)-\frac{1}{4} .
\end{aligned}
$$

Here we consider the dynamical behaviors of the interior equilibrium point $X_{0}^{*}=(2,3,1)$. Other interior equilibrium point of $(5.1)$ can be examined analogously.

For the equilibrium point $X_{0}^{*}(2,3,1)$, we have

$$
x_{0}+\frac{k y_{0}}{x_{0}}=3.5>\frac{m y_{0} E_{0}}{\left(1+m y_{0}\right)^{2}}+\frac{p y_{0} E_{0}}{\left\{\left(1+m y_{0}\right)^{2}\left[(p-c m) y_{0}-c\right]\right\}}=0.375
$$




$$
\begin{aligned}
& k+\frac{k y_{0}}{x_{0}}=2.5>\frac{m x_{0} E_{0}}{\left(1+m y_{0}\right)^{2}}+\frac{p x_{0} E_{0}}{\left\{\left(1+m y_{0}\right)^{2}\left[(p-c m) y_{0}-c\right]\right\}}=0.25, \\
& \widehat{\Delta}=\left\{\left(\frac{k y_{0}}{x_{0}}-\frac{m y_{0} E_{0}}{\left(1+m y_{0}\right)^{2}}-\frac{p y_{0} E_{0}}{\left\{\left(1+m y_{0}\right)^{2}\left[(p-c m) y_{0}-c\right]\right\}}\right)^{2}-x_{0}^{2}-\frac{2 k y_{0}^{2}}{x_{0}}\right\}^{2}-\frac{4 k^{2} y_{0}^{4}}{x_{0}^{2}} \\
& +4\left(k y_{0}-\frac{m x_{0} y_{0} E_{0}}{\left(1+m y_{0}\right)^{2}}-\frac{p x_{0} y_{0} E_{0}}{\left\{\left(1+m y_{0}\right)^{2}\left[(p-c m) y_{0}-c\right]\right\}}\right)^{2}=76.9456>0, \\
& \left\{\frac{k y_{0}}{x_{0}}-\frac{m y_{0} E_{0}}{\left(1+m y_{0}\right)^{2}}-\frac{p y_{0} E_{0}}{\left\{\left(1+m y_{0}\right)^{2}\left[(p-c m) y_{0}-c\right]\right\}}\right\}^{2}=1.2656<x_{0}^{2}+\frac{2 k y_{0}^{2}}{x_{0}}=13, \\
& \frac{k^{2} y_{0}^{2}}{x_{0}^{2}}=2.25>\left\{k-\frac{m x_{0} E_{0}}{\left(1+m y_{0}\right)^{2}}-\frac{p}{\left\{\left(1+m y_{0}\right)^{2}\left[(p-c m) y_{0}-c\right]\right\}}\right\}^{2}=0.5625,
\end{aligned}
$$

so that the Hopf bifurcation conditions of Theorems 2.1,3.1 are satisfied. Besides, since the corresponding equation (2.11) has the form $\omega^{4}-(751 / 64) \omega^{2}+(243 / 16)=0$ and possesses two positive roots $\omega_{+}=3.2020$ and $\omega_{-}=1.2171$, Lemma 2.2 shows that

$$
\begin{aligned}
& \tau_{0}^{+}=\frac{1}{3.2020} \arccos \left(-\frac{162}{\left(64 \cdot(3.2020)^{2}+81\right)}\right)=0.5598, \\
& \tau_{0}^{-}=\frac{1}{1.2171} \arccos \left(-\frac{162}{\left(64 \cdot(1.2171)^{2}+81\right)}\right)=2.2534 .
\end{aligned}
$$

By Theorem $2.1, X_{0}^{*}(2,3,1)$ is locally asymptotically stable if $\tau \in[0,0.5598)$ and unstable if $\tau \in(0.5598,2.2534)$. Therefore, for $\tau_{0}^{+}=0.5598$ the model (5.1) has a Hopf bifurcation. Using Matlab, we also find out that

$$
\begin{aligned}
& c_{1}(0)=0.3537-14.8205 i, \quad \lambda^{\prime}\left(\tau_{0}^{+}\right)=1.8624-2.5129 i, \\
& \mu_{2}=-0.1899<0, \quad \beta_{2}=0.7074>0, \quad T_{2}=8.0019>0 .
\end{aligned}
$$

By Theorem 3.1, the corresponding Hopf bifurcation is subcritical and the bifurcating periodic orbits are unstable and increase.

Theorems $2.1,3.1$ also show that $X_{0}^{*}(2,3,1)$ is locally asymptotically stable for $\tau=$ $0.5498<\tau_{0}^{+}$, consistent with the graphs in Fig. 1 . We also note that for $\tau=\tau_{0}^{+}=0.5598$ there are periodic orbits bifurcating from $X_{0}^{*}(2,3,1)-$ cf. Fig. 2, for $\tau=0.5620>\tau_{0}^{+}$the bifurcating periodic orbits are unstable and increase - cf. Fig. 3, and for $\tau=0.5798>\tau_{0}^{+}$ the equilibrium point $X_{0}^{*}(2,3,1)$ is unstable - cf. Fig. 4 .

Example 5.2 (Singularity-Induced Bifurcation). Using the parameters

$$
a=3, \quad d=2, \quad k=2, \quad m=2, \quad p=3, \quad c=1,
$$

we obtain the following differential-algebra system (4.2):

$$
\begin{aligned}
& \dot{x}(t)=x(t)(3-x(t)-y(t)), \\
& \dot{y}(t)=y(t)\left(2-\frac{2 y(t)}{x(t)}-\frac{E(t)}{1+2 y(t)}\right), \\
& 0=E(t)\left(\frac{3 y(t)}{1+2 y(t)}-1\right)-v .
\end{aligned}
$$



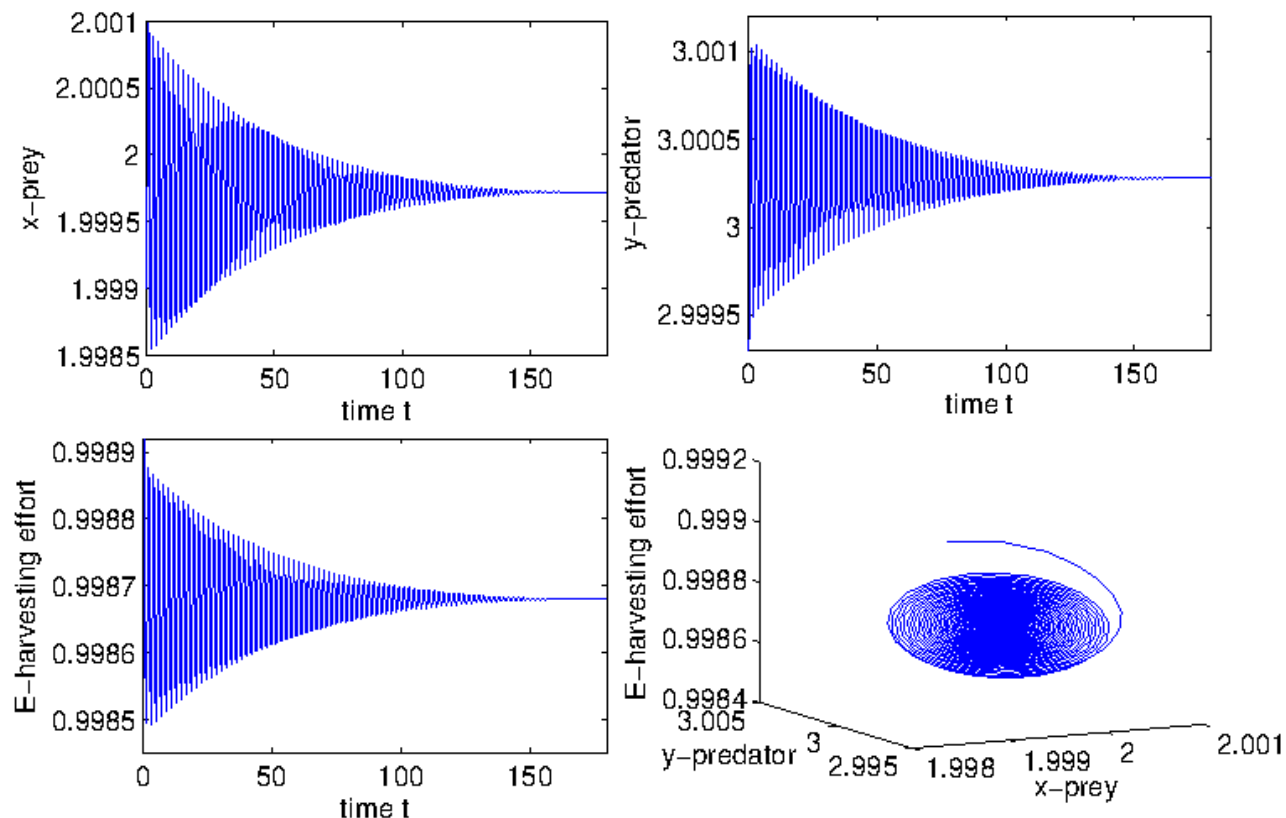

Figure 1: Positive equilibrium point $X_{0}^{*}(2,3,1)$ of system $(5.1)$ is locally asymptotically stable when $\tau=0.5498<\tau_{0}^{+}$. Initial values for numerical simulations are $\left(x_{0}, y_{0}, E_{0}\right)=(1.999,2.999,0.999)$.
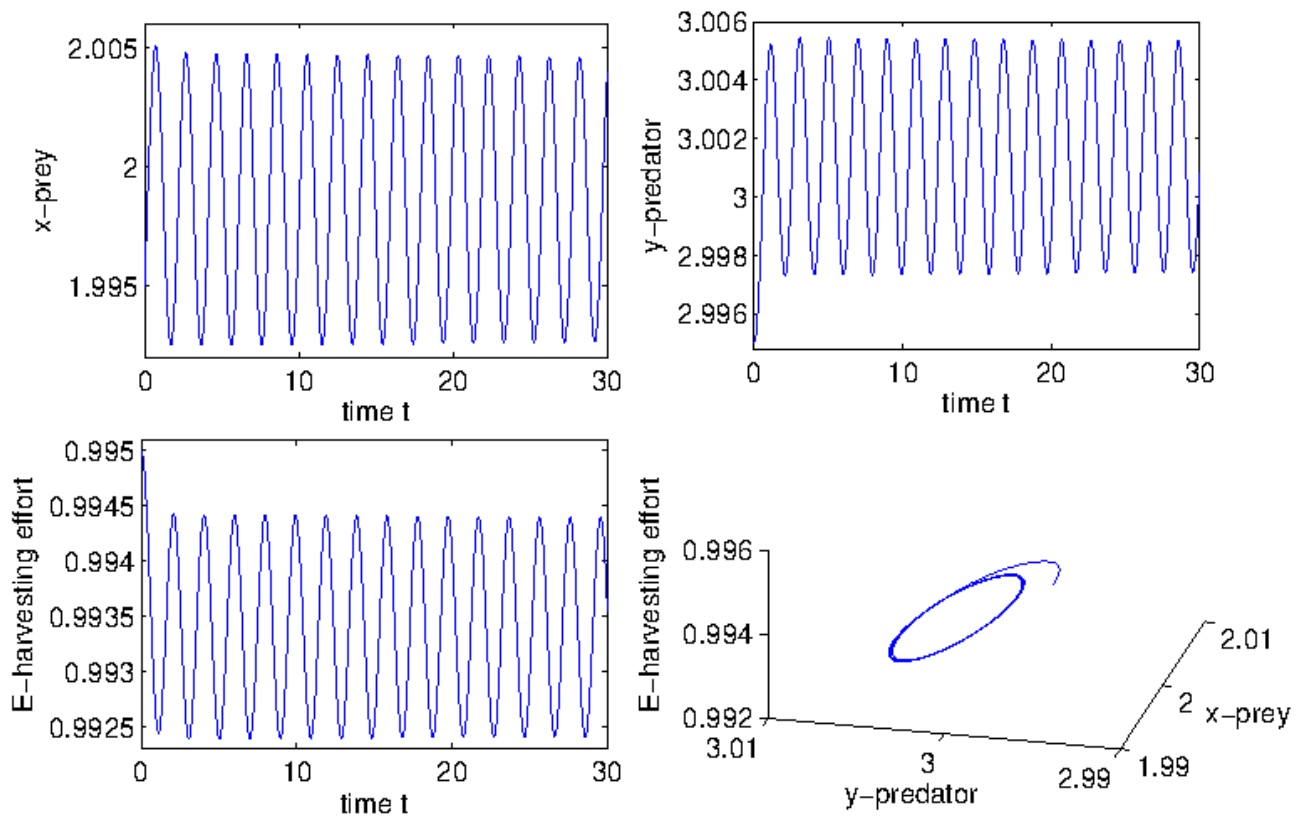

Figure 2: Periodic orbits bifurcating from positive equilibrium point $X_{0}^{*}(2,3,1)$ of $(5.1)$ when $\tau=\tau_{0}^{+}=$ 0.5598. Initial values for numerical simulations are $\left(x_{0}, y_{0}, E_{0}\right)=(1.995,2.995,0.995)$. 

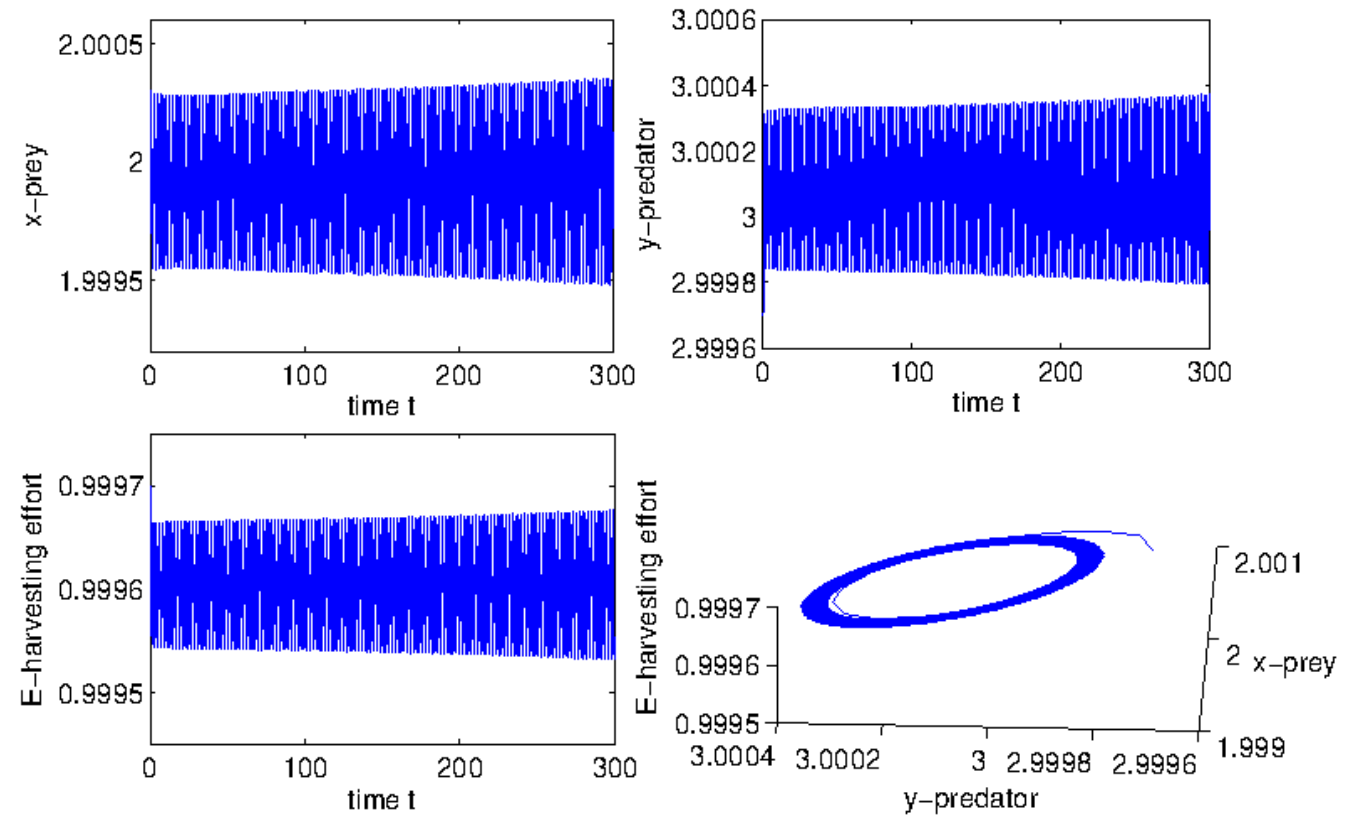

Figure 3: Bifurcating periodic orbits are unstable and increase when $\tau=0.5620>\tau_{0}^{+}$. Initial values for numerical simulations are $\left(x_{0}, y_{0}, E_{0}\right)=(1.9997,2.9997,0.9997)$.
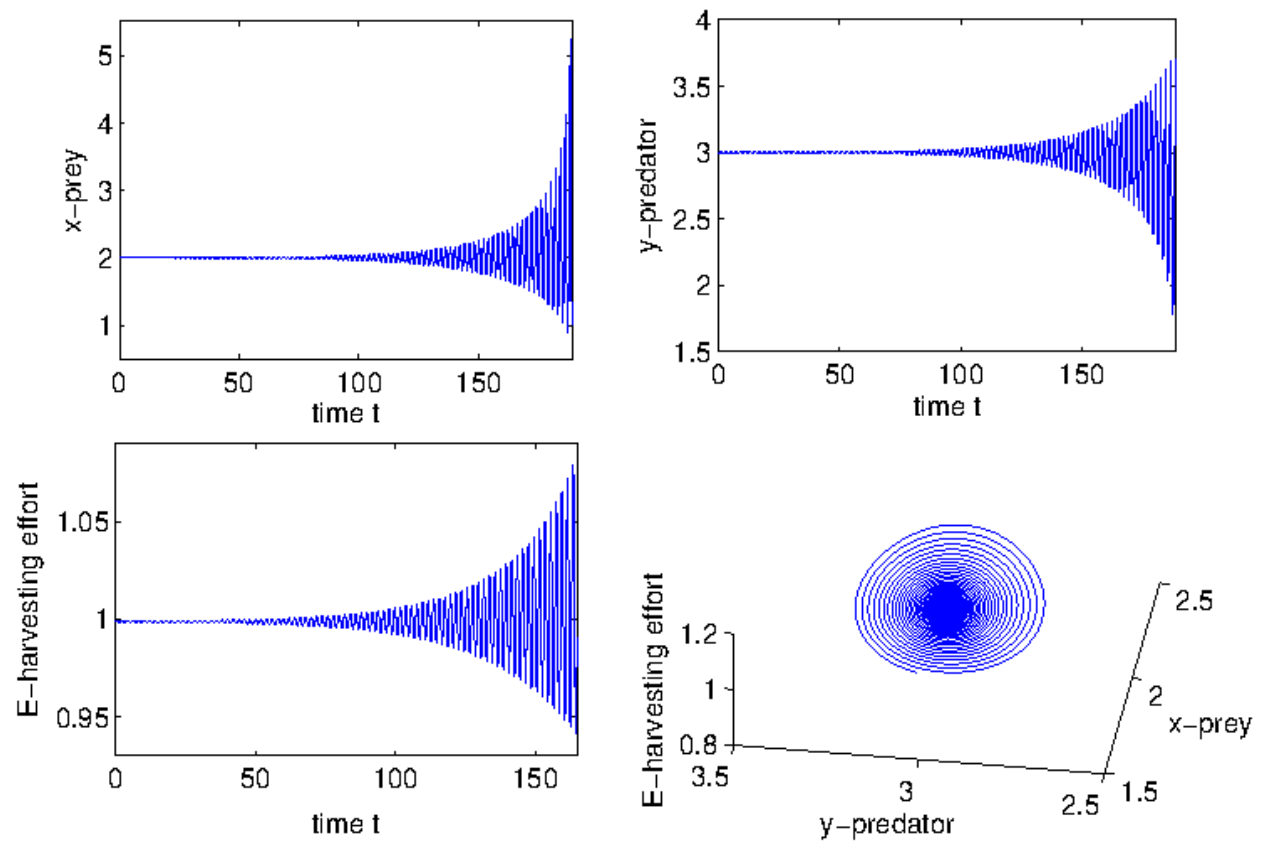

Figure 4: Positive equilibrium point $X_{0}^{*}(2,3,1)$ of $(5.1)$ is unstable when $\tau=0.5798>\tau_{0}^{+}$. Initial values for numerical simulations are $\left(x_{0}, y_{0}, E_{0}\right)=(1.999,2.999,0.999)$. 
This system has the equilibrium point

$$
\left(X_{*}, v_{0}\right)=\left(x_{*}, y_{*}, E_{*}, v_{0}\right)=(2,1,3,0)
$$

and one can check that (5.2) satisfies the conditions SI1)-SI3) of singularity-induced bifurcation. According to Theorem 4.1, this is a singularity-induced bifurcation and there is a stability switch when the harvesting profit $v$ increases through zero. By Theorem 4.2, this singularity-induced bifurcation can be eliminated by the state feedback controller

$$
\bar{u}=\bar{\sigma}\left(E-E_{*}\right)=\bar{\sigma}(E-3) .
$$

The corresponding controlled differential-algebraic system has the form

$$
\begin{aligned}
& \dot{x}(t)=x(t)(3-x(t)-y(t)), \\
& \dot{y}(t)=y(t)\left(2-\frac{2 y(t)}{x(t)}-\frac{E(t)}{1+2 y(t)}\right), \\
& 0=E(t)\left(\frac{3 y(t)}{1+2 y(t)}-1\right)+\bar{\sigma}(E-3)-v .
\end{aligned}
$$

According to (4.4), the feedback gain $\bar{\sigma}$ has to satisfy the inequality $\bar{\sigma}>\max \{1 / 7,2 / 5\}$. Here, we choose $\bar{\sigma}=2$ and by Theorem 4.2 , the controlled differential-algebraic system (5.2) can be stabilised at the equilibrium point $\left(X_{*}, v_{0}\right)=(2,1,3,0)-$ cf. Fig. 5.
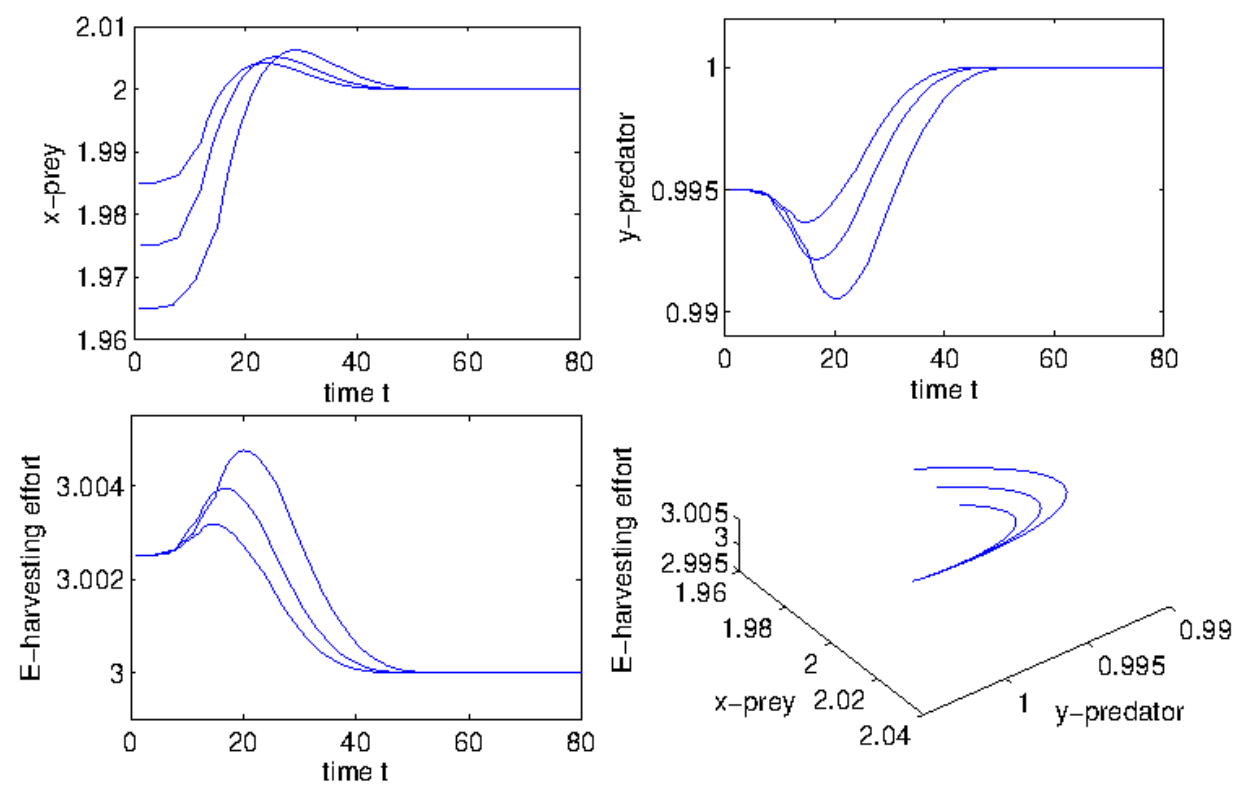

Figure 5: Controlled differential-algebraic system (5.3) is stable at the equilibrium point $\left(X_{*}, v_{0}\right)=$ $(2,1,3,0)$ of differential-algebraic system (5.2). Initial values for numerical simulations are $\left(x_{*}, y_{*}, E_{*}, v_{0}\right)=$ $(1.995-0.01 * k, 0.995,3.0025,0)(k=1,2,3)$. 


\section{Concluding Remarks}

We develop a differential-algebraic predator-prey model with a nonlinear harvesting and gestation delay and study the delay influence on the population dynamics. The parametric procedure of [6] for differential-algebra equations and the results concerning Hopf bifurcation for functional differential equations $[9,17]$ show that the local stability switches of the interior equilibrium point. The dynamical analysis reveals that the Hopf bifurcations occurs when delay increases monotonically through some critical values. For sufficiently small gestation delays, there are conditions providing the stability of interior equilibrium points. However, if delay increases, the stability of the interior equilibrium point mutates into instability for a few times, before becomes unstable for all large delays. Thus the delays play an important role in the stability of interior equilibrium point and can initiate Hopf bifurcations.

In population dynamics, the stability of interior equilibrium points is closely connected to the sustainable development of predator-prey systems. For stable interior equilibrium point, the predators and preys populations and the harvesting effort are in a coexisting equilibrium state less susceptible to outside interference. On the other hand, slightly disturbed unstable interior equilibrium points mean that the predator-prey system can easily lose its ecological equilibrium. Along with the Hopf bifurcations, small-amplitude periodic orbits would bifurcate from the interior equilibrium point - i.e. periodic oscillatory behavior appears and the density of the biological populations and the harvesting effort will fluctuate periodically. Similar to the stability property of interior equilibrium points, the bifurcating periodic orbits are biologically important, since they determine whether the predator and prey populations and the harvesting effort can still coexist in an oscillatory case. Therefore, we also examine the properties of the small-amplitude periodic orbits including direction, stability and period. This helps in better understanding of complicated dynamical phenomena in the harvested predator-prey system.

The singularity-induced bifurcations, also considered here, produce impulse effects in differential-algebra predator-prey systems, usually associated with the rapid growth of species population. With no restrictions present, the species population will grow beyond natural environment carrying capacity that can cause an ecological disbalance. Therefore, preventive measure have to be taken. In order to eliminate singularity-induced bifurcations, we develop a state feedback controller. It is also shown that the singularity-induced bifurcations can be eliminated by intensifying harvesting effort on the predators population. As the result, the ecological balance of the differential-algebra predator-prey system can be restored.

We note that the differential-algebra predator-prey model (1.5) with harvesting is a singular system. Singular systems differ from the systems described by differential equations. Thus singularity-induced bifurcations never appear in familiar harvested predatorprey models described by differential equations - cf. $[2,3,10,35]$. Therefore, the harvested predator-prey model presented here, exposes a richer and more complicated dynamical behavior. It is also worth mentioning, that the parameterisation method transforms the delayed differential-algebra equations of the model (1.5) into delayed differential equa- 
tions (3.9). In a sense, the method is a bridge between differential-algebra and differential equation systems.

(i) The delays due to gestation and maturation of predator population can also be incorporated into model (1.5), and the dynamical effects of multiple delays on the population dynamics could be studied by using the normal form method and center manifold reduction presented in Ref. [11].

(ii) The bifurcations considered here are local, namely, they just exist in the small neighbourhoods of the critical values. So global continuation of the bifurcating periodic orbits in model (1.5) can be further investigated by employing the global Hopf bifurcation theorem developed in Ref. [36].

(iii) Apparently, many stochastic factors in the natural environment can affect the dynamics of biological systems, such as flood, temperature, pestilence, etc. On the grounds of the stochastic modeling methods for population models in Refs. [29,30], the environmental fluctuations can be introduced into model (1.5), and then we can investigate the stochastic stability and bifurcations of the model with stochastic fluctuations and time delay.

We leave the above issues for future consideration.

\section{Acknowledgments}

The authors are greatly indebted to the editor and anonymous referee for careful reading and insightful comments that helped improve this article.

This work is supported by the National Natural Science Foundation of China (Grant No. 11871393), by the Key Project of the International Science and Technology Cooperation Program of Shaanxi Research \& Development Plan (Grant No. 2019KWZ-08), and by the Science and Technology Project founded by the Education Department of Jiangxi Province (Grant No. GJJ14775).

\section{Appendix A}

Here we provide the main points of the parameterisation method [6]. Consider the following differential-algebra system

$$
\begin{aligned}
& \dot{\bar{X}}(t)=f(\bar{X}(t)), \\
& 0=g(\bar{X}(t)),
\end{aligned}
$$

where $f: \mathbb{R}^{n} \rightarrow \mathbb{R}^{n}, g: \mathbb{R}^{n} \rightarrow \mathbb{R}^{\mathrm{r}}$ are continuously differentiable functions,

$$
f:=\left(f_{1}, f_{2}, \ldots, f_{n}\right)^{T}, \quad g:=\left(g_{1}, g_{2}, \ldots, g_{\mathbb{r}}\right)^{T}, \quad n>\mathbb{r} .
$$

Suppose that $\bar{X}_{0}$ is an equilibrium point of (A.1). 
If (A.1) satisfies the conditions

$$
\operatorname{rank} D_{\bar{X}} g\left(\bar{X}_{0}\right)=\mathrm{r}, \quad D_{\bar{X}} g\left(\bar{X}_{0}\right)=(0, P)_{\mathrm{r} \times n},
$$

where $P_{\mathrm{r} \times \mathrm{r}}$ is a nonsingular matrix, then the system (A.1) can be parameterised as follows:

$$
\begin{aligned}
& \bar{X}(t)=\psi(Y(t))=\bar{X}_{0}+U_{0} Y(t)+V_{0} h(Y(t)), \\
& g(\psi(Y(t)))=0,
\end{aligned}
$$

where

$$
Y:=\left(y_{1}, y_{2}, \ldots, y_{n-\mathrm{r}}\right)^{T} \in \mathbb{R}^{(n-\mathrm{r})}, \quad U_{0}=\left(\begin{array}{c}
I_{(n-\mathrm{r})} \\
0
\end{array}\right)_{n \times(n-\mathrm{r})}, \quad V_{0}=\left(\begin{array}{c}
0 \\
I_{\mathrm{r}}
\end{array}\right)_{n \times \mathrm{r}},
$$

$I_{\mathrm{r}}$ is the identity $\mathbb{r} \times \mathbb{r}$ matrix and $h(Y)$ a smooth mapping from $\mathbb{R}^{(n-\mathrm{r})}$ to $\mathbb{R}^{\mathrm{r}}$. Substituting $\bar{X}(t)=\psi(Y(t))$ in the first equation of (A.1) yields

$$
D_{Y} \psi(Y(t)) \dot{Y}(t)=f(\psi(Y(t))) .
$$

Differentiating (A.2) and (A.3) with respect to $Y$ and multiplying the first derivative by $U_{0}^{T}$ from the left, we obtain

$$
\begin{aligned}
& U_{0}^{T} D_{Y} \psi(Y(t))=I_{(n-r)}, \\
& D_{\bar{X}} g(\bar{X}(t)) D_{Y} \psi(Y(t))=0 .
\end{aligned}
$$

The Eqs. (A.4)-(A.6) yield that

$$
\left(\begin{array}{c}
D_{\bar{X}} g(\bar{X}(t)) \\
U_{0}^{T}
\end{array}\right)^{-1}\left(\begin{array}{c}
0 \\
I_{(n-r)}
\end{array}\right) \dot{Y}(t)=f(\psi(Y(t))),
$$

and, consequently,

$$
\left(\begin{array}{c}
0 \\
I_{(n-r)}
\end{array}\right) \dot{Y}(t)=\left(\begin{array}{c}
0 \\
U_{0}^{T} f(\psi(Y(t)))
\end{array}\right)
$$

so that system (A.1) is equivalent to the parameterised system

$$
\dot{Y}(t)=U_{0}^{T} f(\psi(Y(t))) \text {. }
$$

\section{Appendix B}

Let us show how to establish the normal form (3.1). First, we write the Taylor expansions of the parameterised system (2.4) as

$$
\begin{aligned}
\dot{y}_{1}= & f_{1 y_{1}}\left(\bar{X}_{0}\right) y_{1}+f_{1 y_{2}}\left(\bar{X}_{0}\right) y_{2}+\frac{1}{2} f_{1 y_{1} y_{1}}\left(\bar{X}_{0}\right) y_{1}^{2}+f_{1 y_{1} y_{2}}\left(\bar{X}_{0}\right) y_{1} y_{2} \\
& +\frac{1}{2} f_{1 y_{2} y_{2}}\left(\bar{X}_{0}\right) y_{2}^{2}+\mathscr{O}\left(|Y|^{3}\right), \\
\dot{y}_{2}= & f_{2 y_{1}}\left(\bar{X}_{0}\right) y_{1}+f_{2 y_{2}}\left(\bar{X}_{0}\right) y_{2}+\frac{1}{2} f_{2 y_{1} y_{1}}\left(\bar{X}_{0}\right) y_{1}^{2}+f_{2 y_{1} y_{2}}\left(\bar{X}_{0}\right) y_{1} y_{2} \\
& +\frac{1}{2} f_{2 y_{2} y_{2}}\left(\bar{X}_{0}\right) y_{2}^{2}+\mathscr{O}\left(|Y|^{3}\right),
\end{aligned}
$$


and determine the coefficients in the above expansions. In follow from (2.3) that

$$
\begin{aligned}
D_{\bar{X}} f_{1}(\bar{X})= & \left(a-x(t-\tau)-x e^{-\lambda \tau}-y,-x, 0\right), \\
D_{\bar{X}} f_{2}(\bar{X})= & \left(\frac{k y^{2}}{x^{2}}, d-\frac{2 k y}{x}-\frac{\bar{E}}{1+m y}+\frac{m y \bar{E}}{(1+m y)^{2}}\right. \\
& +\frac{p E_{0} y}{\left(1+m y_{0}\right)\left[(p-c m) y_{0}-c\right](1+m y)} \\
& \left.+\frac{p E_{0} y}{\left(1+m y_{0}\right)\left[(p-c m) y_{0}-c\right](1+m y)^{2}},-\frac{y}{1+m y}\right), \\
D_{\bar{X}} g(\bar{X})= & \left(0, \frac{p \bar{E}}{(1+m y)^{2}}-\frac{p^{2} E_{0} y}{\left(1+m y_{0}\right)\left[(p-c m) y_{0}-c\right](1+m y)^{2}}\right. \\
& \left.-\frac{p E_{0}[(p-c m) y-c]}{\left(1+m y_{0}\right)\left[(p-c m) y_{0}-c\right](1+m y)}, \frac{(p-c m) y-c}{1+m y}\right) .
\end{aligned}
$$

Recalling the Eqs. (A.5) and (A.6), we obtain

$$
D_{Y} \psi(Y)=\left(\begin{array}{c}
D_{\bar{X}} g(\bar{X}) \\
U_{0}^{T}
\end{array}\right)^{-1}\left(\begin{array}{c}
0 \\
I_{2}
\end{array}\right)=\left(\begin{array}{cc}
1 & 0 \\
0 & 1 \\
0 & -\frac{(1+m y) \varrho(\bar{X})}{(p-c m) y-c}
\end{array}\right):=\left(D_{y_{1}} \psi(Y), D_{y_{2}} \psi(Y)\right),
$$

where

$$
\begin{aligned}
\varrho(\bar{X})= & \frac{p \bar{E}}{(1+m y)^{2}}-\frac{p^{2} E_{0} y}{\left\{\left(1+m y_{0}\right)\left[(p-c m) y_{0}-c\right](1+m y)^{2}\right\}} \\
& -\frac{p E_{0}[(p-c m) y-c]}{\left\{\left(1+m y_{0}\right)\left[(p-c m) y_{0}-c\right](1+m y)\right\}}
\end{aligned}
$$

Besides,

$$
\begin{aligned}
\varrho\left(\bar{X}_{0}\right)= & {\left[\frac{p}{\left(1+m y_{0}\right)^{2}}\right]\left\{E_{0}+\frac{p y_{0} E_{0}}{\left\{\left(1+m y_{0}\right)\left[(p-c m) y_{0}-c\right]\right\}}\right\} } \\
& -\frac{p^{2} y_{0} E_{0}}{\{P(m, c)\}}-\frac{p E_{0}}{\left(1+m y_{0}\right)^{2}}=0 .
\end{aligned}
$$

The Eqs. (2.4),(B.2) and (B.3) yield

$$
\begin{aligned}
f_{1 y_{1}}(\bar{X})= & D_{\bar{X}} f_{1}(\bar{X}) D_{y_{1}} \psi(Y)=a-x(t-\tau)-x e^{-\lambda \tau}-y, \\
f_{1 y_{2}}(\bar{X})= & D_{\bar{X}} f_{1}(\bar{X}) D_{y_{2}} \psi(Y)=-x, \quad f_{2 y_{1}}(\bar{X})=D_{\bar{X}} f_{2}(\bar{X}) D_{y_{1}} \psi(Y)=\frac{k y^{2}}{x^{2}}, \\
f_{2 y_{2}}(\bar{X})= & D_{\bar{X}} f_{2}(\bar{X}) D_{y_{2}} \psi(Y)=d-\frac{2 k y}{x}-\frac{\bar{E}}{1+m y}+\frac{p E_{0} y}{\left(1+m y_{0}\right)\left[(p-c m) y_{0}-c\right](1+m y)} \\
& +\frac{p E_{0} y}{\left(1+m y_{0}\right)\left[(p-c m) y_{0}-c\right](1+m y)^{2}}+\frac{m y \bar{E}}{(1+m y)^{2}}-\frac{y}{(p-c m) y-c} \\
& \times\left\{\frac{p^{2} E_{0} y}{\left(1+m y_{0}\right)\left[(p-c m) y_{0}-c\right](1+m y)^{2}}\right.
\end{aligned}
$$




$$
\left.+\frac{p E_{0}[(p-c m) y-c]}{\left(1+m y_{0}\right)\left[(p-c m) y_{0}-c\right](1+m y)}-\frac{p \bar{E}}{(1+m y)^{2}}\right\},
$$

and substituting $\bar{X}_{0}$ into (B.4) shows that

$$
\begin{array}{ll}
f_{1 y_{1}}\left(\bar{X}_{0}\right)=-x_{0} e^{-\lambda \tau}, & f_{1 y_{2}}\left(\bar{X}_{0}\right)=-x_{0}, \\
f_{2 y_{1}}\left(\bar{X}_{0}\right)=\frac{k y_{0}^{2}}{x_{0}^{2}}, & f_{2 y_{2}}\left(\bar{X}_{0}\right)=-\mathscr{M} .
\end{array}
$$

Invoking (B.4) again, we obtain

$$
\begin{aligned}
D_{\bar{X}} f_{1 y_{1}}(\bar{X})= & \left(-2 e^{-\lambda \tau},-1,0\right), \quad D_{\bar{X}} f_{1 y_{2}}(\bar{X})=(-1,0,0) \\
D_{\bar{X}} f_{2 y_{1}}(\bar{X})= & \left(-\frac{2 k y^{2}}{x^{3}}, \frac{2 k y}{x^{2}}, 0\right), \\
D_{\bar{X}} f_{2 y_{2}}(\bar{X})= & \left(\frac{2 k y}{x^{2}},-\frac{2 k}{x}+\frac{2 m \bar{E}}{(1+m y)^{2}}-\frac{2 m^{2} y \bar{E}}{(1+m y)^{3}}\right. \\
& +\frac{2 p E_{0}}{\left(1+m y_{0}\right)\left[(p-c m) y_{0}-c\right](1+m y)^{2}} \\
& -\frac{2 p m E_{0} y}{\left(1+m y_{0}\right)\left[(p-c m) y_{0}-c\right](1+m y)^{3}}+\frac{c}{[(p-c m) y-c]^{2}} \\
& \times\left[\frac{p^{2} E_{0} y}{\left(1+m y_{0}\right)\left[(p-c m) y_{0}-c\right](1+m y)^{2}}\right. \\
& \left.+\frac{p E_{0}[(p-c m) y-c]}{\left(1+m y_{0}\right)\left[(p-c m) y_{0}-c\right](1+m y)}-\frac{p \bar{E}}{(1+m y)^{2}}\right]-\frac{2 p^{2} E_{0}}{(p-c m) y-c} \\
& \times\left[\frac{2 y^{2}}{\left(1+m y_{0}\right)\left[(p-c m) y_{0}-c\right](1+m y)^{2}}\right. \\
& \left.-\frac{2 p^{2} m E_{0} y}{\left(1+m y_{0}\right)\left[(p-c m) y_{0}-c\right](1+m y)^{3}}+\frac{2 p m \bar{E}}{(1+m y)^{3}}\right] \\
& \left.-\frac{1}{(1+m y)^{2}}+\frac{p y}{(1+m y)^{2}[(p-c m) y-c]}\right) .
\end{aligned}
$$

We now substitute $\bar{X}_{0}$ into the Eqs. (B.3),(B.6) to obtain

$$
\begin{aligned}
D_{\bar{X}} f_{1 y_{1}}\left(\bar{X}_{0}\right)= & \left(-2 e^{-\lambda \tau},-1,0\right), \quad D_{\bar{X}} f_{1 y_{2}}\left(\bar{X}_{0}\right)=(-1,0,0) \\
D_{\bar{X}} f_{2 y_{1}}\left(\bar{X}_{0}\right)= & \left(-\frac{2 k y_{0}^{2}}{x_{0}^{3}}, \frac{2 k y_{0}}{x_{0}^{2}}, 0\right), \\
D_{\bar{X}} f_{2 y_{2}}\left(\bar{X}_{0}\right)= & \left(\frac{2 k y_{0}}{x_{0}^{2}}, \frac{2 m E_{0}}{\left(1+m y_{0}\right)^{3}}+\frac{2 p E_{0}}{P(m, c)}-\frac{2 p m y_{0} E_{0}}{P(m, c)}-\frac{2 p^{2} y_{0} E_{0}}{Q(m, c)}-\frac{2 k}{x_{0}},\right. \\
& \left.-\frac{1}{\left(1+m y_{0}\right)^{2}}+\frac{p y_{0}}{\left(1+m y_{0}\right)^{2}\left[(p-c m) y_{0}-c\right]}\right)
\end{aligned}
$$




$$
D_{Y} \psi(0)=\left(D_{y_{1}} \psi(0), D_{y_{2}} \psi(0)\right)=\left(\begin{array}{cc}
1 & 0 \\
0 & 1 \\
0 & -\frac{\left(1+m y_{0}\right) \varrho\left(\bar{X}_{0}\right)}{(p-c m) y_{0}-c}
\end{array}\right)=\left(\begin{array}{ll}
1 & 0 \\
0 & 1 \\
0 & 0
\end{array}\right) .
$$

It follows from (2.4),(B.7) that

$$
\begin{aligned}
f_{1 y_{1} y_{1}}\left(\bar{X}_{0}\right)= & D_{\bar{X}} f_{1 y_{1}}\left(\bar{X}_{0}\right) D_{y_{1}} \psi(0)=-2 e^{-\lambda \tau} \\
f_{1 y_{1} y_{2}}\left(\bar{X}_{0}\right)= & D_{\bar{X}} f_{1 y_{1}}\left(\bar{X}_{0}\right) D_{y_{2}} \psi(0)=-1, \\
f_{1 y_{2} y_{2}}\left(\bar{X}_{0}\right)= & D_{\bar{X}} f_{1 y_{2}}\left(\bar{X}_{0}\right) D_{y_{2}} \psi(0)=0, \\
f_{2 y_{1} y_{1}}\left(\bar{X}_{0}\right)= & D_{\bar{X}} f_{2 y_{1}}\left(\bar{X}_{0}\right) D_{y_{1}} \psi(0)=-\frac{2 k y_{0}^{2}}{x_{0}^{3}}, \\
f_{2 y_{1} y_{2}}\left(\bar{X}_{0}\right)= & D_{\bar{X}} f_{2 y_{1}}\left(\bar{X}_{0}\right) D_{y_{2}} \psi(0)=\frac{2 k y_{0}}{x_{0}^{2}}, \\
f_{2 y_{2} y_{2}}\left(\bar{X}_{0}\right)= & D_{\bar{X}} f_{2 y_{2}}\left(\bar{X}_{0}\right) D_{y_{2}} \psi(0)=\frac{2 m E_{0}}{\left(1+m y_{0}\right)^{3}} \\
& +\frac{2 p E_{0}}{P(m, c)}-\frac{2 p m y_{0} E_{0}}{P(m, c)}-\frac{2 p^{2} y_{0} E_{0}}{Q(m, c)}-\frac{2 k}{x_{0}}
\end{aligned}
$$

and substituting the coefficients (B.5) and (B.8) into (B.1) leads to (3.1).

\section{Appendix C}

In a differential-algebra systems, singularity-induced bifurcations appear when the equilibrium crosses a singular surface of the system at a bifurcation point. If the trajectory of the system crosses singularity surface in a finite time with infinite speed, the system changes its stability status. Such bifurcations can be described by the singularity-induced bifurcation theorem from [35], which is recalled here.

Consider a differential-algebraic system with a one-dimensional parameter $\mu$, viz.

$$
\begin{aligned}
& \dot{X}(t)=f(x(t), y(t), \mu), \\
& 0=g(x(t), y(t), \mu),
\end{aligned}
$$

where

$$
X(t):=(x(t), y(t))^{T}, \quad x(t) \in \bar{X} \subset \mathbb{R}^{n}, \quad y(t) \in \bar{Y} \subset \mathbb{R}^{m}, \quad \mu \in \bar{\Lambda} \subset \mathbb{R}^{q},
$$

$f: \mathbb{R}^{m+n+q} \rightarrow \mathbb{R}^{n}, g: \mathbb{R}^{m+n+q} \rightarrow \mathbb{R}^{m}$, and $n, m, q$ are positive integers. The equation $g(x, y, \mu)=0$ is a constraint condition for the system (C.1).

Set

$$
\Delta(x, y, \mu):=\operatorname{det}\left(D_{y} g(x, y, \mu)\right) .
$$

We assume that $\left(x_{0}, y_{0}, \mu_{0}\right)$ is an equilibrium point of (C.1) and the system (C.1) satisfies the following conditions: 
SI1) $\left.D_{y} g(x, y, \mu)\right|_{\left(x_{0}, y_{0}, \mu_{0}\right)}$ has a simple zero eigenvalue, and $\operatorname{trace}\left(D_{y} f \operatorname{adj}\left(D_{y} g\right) D_{x} g\right)$ is not equal to zero at $\left(x_{0}, y_{0}, \mu_{0}\right)$.

SI2) $\left(\begin{array}{ll}D_{x} f & D_{y} f \\ D_{x} g & D_{y} g\end{array}\right)$ is nonsingular at $\left(x_{0}, y_{0}, \mu_{0}\right)$.

SI3) $\left(\begin{array}{ccc}D_{x} f & D_{y} f & D_{\mu} f \\ D_{x} g & D_{y} g & D_{\mu} g \\ D_{x} \Delta & D_{y} \Delta & D_{\mu} \Delta\end{array}\right)$ is also nonsingular at $\left(x_{0}, y_{0}, \mu_{0}\right)$.

Then there exists a smooth equilibrium curve of differential-algebraic system (C.1), which passes through $\left(x_{0}, y_{0}, \mu_{0}\right)$ and is transversal to the singular surface

$$
S=\{(x, y, \mu) \in \bar{X} \times \bar{Y} \times \bar{\Lambda}: g(x, y, \mu)=0, \Delta(x, y, \mu)=0\} .
$$

On the singular surface $S$ there is a point, called singular point of the system (C.1), which plays a significant role in the occurrence of singularity-induced bifurcation. For $\mu=\mu_{0}$, one eigenvalue of the system (C.1) moves along the real axis from $\mathbb{C}^{-}$to $\mathbb{C}^{+}$by diverging through $\infty$ if $\bar{M} / \bar{N}>0$ or from $\mathbb{C}^{+}$to $\mathbb{C}^{-}$if $\bar{M} / \bar{N}<0$, where

$$
\begin{aligned}
\bar{M} & :=-\left.\operatorname{trace}\left(D_{y} f \operatorname{adj}\left(D_{y} g\right) D_{x} g\right)\right|_{\left(x_{0}, y_{0}, \mu_{0}\right)} \\
\bar{N} & :=\left(D_{\mu} \Delta-\left(D_{x} \Delta, D_{y} \Delta\right)\left(\begin{array}{cc}
D_{x} f & D_{y} f \\
D_{x} g & D_{y} g
\end{array}\right)^{-1}\left(\begin{array}{c}
D_{\mu} f \\
D_{\mu} g
\end{array}\right)\right)_{\left(x_{0}, y_{0}, \mu_{0}\right)} .
\end{aligned}
$$

The change of the sign of the eigenvalue changes the stability status of system (C.1). The remaining $(n-1)$ eigenvalues are bounded and stay away from the origin. Thus the singularity-induced bifurcation is the change of the stability status of the differentialalgebraic system (C.1) because one eigenvalue of (C.1) diverges to infinity when the Jacobian $\left.D_{y} g(x, y, \mu)\right|_{\left(x_{0}, y_{0}, \mu_{0}\right)}$ is singular.

\section{References}

[1] S. Ayasun, C.O. Nwankpa and H.G. Kwatny, Computation of singular and singularity induced bifurcation points of differential-algebraic power system model, IEEE Trans. Circuits Syst. I. Regul. Pap. 51, 1525-1538 (2004).

[2] R.E. Beardmore, The singularity induced bifurcation and its Kronecker normal form, SIAM J. Matrix Anal. Appl. 23, 126-137 (2001).

[3] R.E. Beardmore and R. Laister, The flow of a differential-algebraic equation near a singular equilibrium, SIAM J. Matrix Anal. Appl. 24, 106-120 (2002).

[4] W.M. Boothby, An Introduction to differential manifolds and Riemannian geometry, Academic Press (1986).

[5] B.S. Chen and J.J. Chen, Bifurcation and chaotic behavior of a discrete singular biological economic system, Appl. Math. Comput. 219, 2371-2386 (2012).

[6] B.S. Chen, X.X. Liao and Y.Q. Liu, Normal forms and bifurcations for the differential-algebraic systems (in Chinese), Acta Math. Appl. Sin. 23, 429-443 (2000). 
[7] L.S. Chen, Mathematical models and methods in ecology (in Chinese), Science Press (2017).

[8] C.W. Clark, Mathematical bioeconomics: The mathematics of conservation, Wiley (2010).

[9] K.L. Cooke and Z. Grossman, Discrete delay, distributed delay and stability switches, J. Math. Anal. Appl. 86, 592-627 (1982).

[10] L. Dai, Singular control system, Springer (1989).

[11] T. Faria and L.T. Maglhalães, Normal form for retarded functional differential equations with parameters and applications to Hopf bifurcation, J. Differential Equations 122, 181-200 (1995).

[12] H.I. Freedman, V.S. Harirao and K.J. Lakshmi, Recent trends in differential equations, World Scientific (1992).

[13] K. Gopalsamy, Stability and oscillations in delay differential equations of population dynamics, Kluwer Academic (1992).

[14] H.S. Gordon, Economic theory of a common property resource: the fishery, J. Polit. Econ. 62, 124-142 (1954).

[15] J. Guckenheimer and P. Holmes, Nonlinear oscillations, dynamical systems, and bifurcations of vector fields, Springer (1983).

[16] Z. Gui and W. Ge, The effect of harvesting on a predator-prey system with stage structure, Ecol. Model. 187, 329-340 (2005).

[17] J. Hale and S.V. Lunel, Introduction to functional differential equations, Springer (1993).

[18] B. Hassard, D. Kazarinoff and Y. Wan, Theory and applications of Hopf bifurcation, Cambridge University Press (1981).

[19] C.M. Heggerud and K.Q. Lan, Local stability analysis of ratio-dependent predator-prey models with predator harvesting rates, Appl. Math. Comput. 270, 349-357 (2015).

[20] A. Ilchmann and T. Reis (Eds.), Surveys in differential-algebraic equations I, Springer (2013).

[21] T.K. Kar and U.K. Pahari, Non-selective harvesting in prey-predator models with delay, Commun. Nonlinear Sci. Numer. Simul. 11, 499-509 (2006).

[22] Y. Kuang, Delay differential equations with applications in population dynamics, Academic Press (1993).

[23] P.H. Leslie and J.C. Gower, The properties of a stochastic model for the predator-prey type of interaction between two species, Biometrika 47, 219-234 (1960).

[24] M. Li, B.S. Chen and H.W. Ye, A bioeconomic differential algebraic predator-prey model with nonlinear prey harvesting, Appl. Math. Model. 42, 17-28 (2017).

[25] W. Liu and Y.L. Jiang, Nonlinear dynamical behaviour in a predator-prey model with harvesting, East Asian J. Appl. Math. 7, 376-395 (2017).

[26] W. Liu and Y.L. Jiang, Bifurcation in a differential-algebra predator-prey system with time lag effects, East Asian J. Appl. Math. 9, 122-152 (2019).

[27] W. Marszalek and Z.W. Trzaska, Singularity-induced bifurcations in electrical power systems, IEEE Trans. Power Syst. 20, 312-320 (2005).

[28] A.A. Martynyuk, Stability theory for dynamic equations on time scales, Springer (2016).

[29] B. Mukhopadhyay and R. Bhattacharyya, Role of gestation delay in a plankton-fish model under stochastic fluctuations, Math. Biosci. 215, 26-34 (2008).

[30] R.M. Nisbet and W.S.C. Gurney, Modelling fluctuating populations, Wiley (1982).

[31] S. Reich, On the local qualitative behavior of differential-algebraic equations, Circuits Systems Signal Process. 14, 427-443 (1995).

[32] R. Riaza, Singularity-induced bifurcations in lumped circuits, IEEE Trans. Circuits Syst. I. Regul. Pap. 52, 1442-1450 (2005).

[33] S. Ruan and J. Wei, On the zeros of transcendental functions with applications to stability of delay differential equations with two delays, Dyn. Contin. Discrete Impuls. Syst. Ser. A Math. Anal. 10, 863-874 (2003). 
[34] H.R. Thieme, Mathematics in population biology, Princeton University Press (2003).

[35] V. Venkatasubramanian, H. Schättler and J. Zaborszky, Local bifurcation and feasibility regions in differential-algebraic systems, IEEE Trans. Automat. Control 40, 1992-2013 (1995).

[36] J. Wu, Symmetric functional differential equations and neural networks with memory, Trans. Amer. Math. Soc. 350, 4799-4838 (1998).

[37] Y.X. Xu and M.Y. Huang, Homoclinic orbits and Hopf bifurcations in delay differential systems with T-B singularity, J. Differential Equations 244, 582-598 (2008).

[38] L.J. Yang and Y. Tang, An improved version of the singularity induced bifurcation theorem, IEEE Trans. Automat. Control 49, 1483-1486 (2001).

[39] Z.G. Zeng, P. Yu and X.X. Liao, A new comparison method for stability theory of differential systems with time-varying delays, Internat. J. Bifur. Chaos Appl. Sci. Engrg. 18, 169-186 (2008).

[40] Z.F. Zhang, T.R. Ding, W.Z. Huang and Z.X. Dong, Qualitative theory of differential equations (in Chinese), Science Press (1985).

[41] J.Y. Zhang and B.Y. Feng, Geometry theory and bifurcation problems of ordinary differential equations (in Chinese), Peking University Press (2000).

[42] G.D. Zhang, J. Hu and Z. Zeng, New criteria on global stabilization of delayed memristive neural networks with inertial item, IEEE Trans. Cybern. In press, On-line vr. DOI:10.1109/TCYB.2018.2889653 (2019).

[43] G.D. Zhang and Y. Shen, Periodic solutions for a neutral delay Hassell-Varley type predator-prey system, Appl. Math. Comput. 264, 443-452 (2015).

[44] G.D. Zhang, Y. Shen and B.S. Chen, Hopf bifurcation of a predator-prey system with predator harvesting and two delays, Nonlinear Dyn. 73, 2119-2131 (2013).

[45] G.D. Zhang, Y. Shen and B.S. Chen, Bifurcation analysis in a discrete differential-algebraic predator-prey system, Appl. Math. Model. 38, 4835-4848 (2014).

[46] G.D. Zhang, Z. Zeng and J. Hu, New results on global exponential dissipativity analysis of memristive inertial neural networks with distributed time-varying delays, Neural Netw. 97, 183-191 (2018). 